\title{
HIGHER ORDER DERIVED FUNCTORS AND THE ADAMS SPECTRAL SEQUENCE
}

\author{
HANS-JOACHIM BAUES AND DAVID BLANC
}

\begin{abstract}
Classical homological algebra studies chain complexes, resolutions, and derived functors in additive categories. In this paper we define higher order chain complexes, resolutions, and derived functors in the context of a new type of algebraic structure, called an algebra of left cubical balls. We show that higher order resolutions exist in these algebras, and that they determine higher order Ext-groups. In particular, the $E_{m}$-term of the Adams spectral sequence $(m>2)$ is such a higher Ext-group, providing a new way of constructing its differentials.
\end{abstract}

\section{INTRODUCTION}

Topologists have been working on the problem of calculating the homotopy groups of spheres for around eighty years, and many methods have been developed for this purpose. One of the most useful tools for this purpose is the Adams spectral sequence $E_{2}, E_{3}, E_{4}, \ldots$, converging to the $p$-completed stable homotopy groups of the sphere. Adams computed the $E_{2}$-term of the spectral sequence, and showed that it is algebraically determined:

$$
E_{2}^{s, t} \cong \operatorname{Ext}_{\mathcal{A}}^{s, t}\left(\mathbb{F}_{p}, \mathbb{F}_{p}\right)
$$

where the derived functor Ext is taken for modules over the $\bmod p$ Steenrod algebra $\mathcal{A}$ of primary mod $p$ cohomology operations (cf. [A]).

Elements in the mod $p$ cohomology $H^{n}\left(X ; \mathbb{F}_{p}\right)$ of a space $X$ are given by homotopy classes of maps from $X$ to an Eilenberg-Mac Lane space $K\left(\mathbb{F}_{p}, n\right)$, and the elements of the Steenrod algebra are homotopy classes of maps between such Eilenberg-Mac Lane spaces, acting on cohomology classes by composition.

This structure suffices to recover the $E_{2}$-term of the Adams spectral sequence, by (0.1). However, in order to determine the higher terms $E_{3}, E_{4}, \ldots$, we have to look not only at homotopy classes of maps, but at the space $\operatorname{Map}\left(X, K\left(\mathbb{F}_{p}, n\right)\right)$ of all maps from $X$ to $K\left(\mathbb{F}_{p}, n\right)$, together with the action of the mapping spaces $\operatorname{Map}\left(K\left(\mathbb{F}_{p}, n\right), K\left(\mathbb{F}_{p}, k\right)\right)$ on it. This structure is called the Eilenberg-Mac Lane mapping algebra of $X$ (cf. [BB1]).

As we show in this paper, this mapping algebra suffices to compute the whole Adams spectral sequence (cf. [BB2]). However, this structure is still too complicated for computational purposes, because it involves the topology of the mapping spaces, and is not algebraic in nature. Therefore, our main aim here is to extract from these spaces the appropriate algebraic data needed to calculate all the differentials.

Date: June 28, 2018.

1991 Mathematics Subject Classification. Primary: 55T15; secondary: 18G40, 18G50, 55S20.

Key words and phrases. Adams spectral sequence, higher chain complexes, higher cohomology operations, higher Ext-groups, higher order resolutions, left cubical balls. 
For this purpose, we consider singular cubes $\sigma: I^{n} \rightarrow \operatorname{Map}(X, Y)$ in the above mapping spaces. Appropriate collections of such cubes can be glued together to form a left cubical ball, as in Figure1 (see $\$ 9$ below). Such balls have a new kind of combinatorialalgebraic structure (coming from pasting operations, and so on), which we call an algebra of left $n$-cubical balls. The case $n=1$ is discussed in detail in Section 12, where we show that an algebra of left 1-cubical balls corresponds to the notion of an abelian track category.

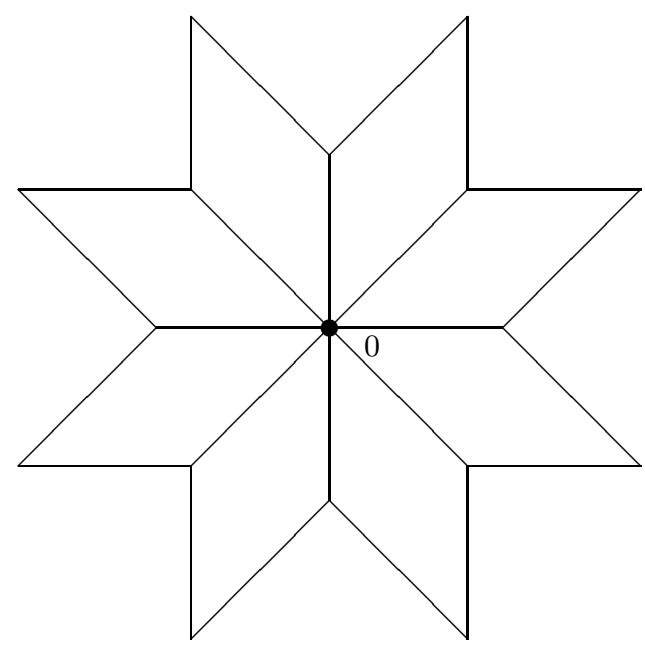

Figure 1. A left 2-cubical ball

In such algebras, we define the notion of a higher order chain complex, replacing the equation $\partial_{n-1} \circ \partial_{n}=0$ in an ordinary chain complex by nullhomotopies $H_{n}$ : $\partial_{n-1} \circ \partial_{n} \sim 0$, and higher nullhomotopies (see Section 31). This allows us to define higher order resolutions, in Section 8, and show:

Theorem A. Higher order resolutions exist in any algebra of left $n$-cubical balls.

See Theorem 14.5 below.

It turns out that such higher order resolutions provide a method for calculating the higher terms of the Adams spectral sequence, which thus may be identified as certain $m$-th order derived functors, called $m$-th order Ext-groups:

Theorem B. Any $n$-th order resolution determines the higher order Ext-groups $E_{m}$ for $m \leq n+2$. In the algebra of left cubical balls determined by the Eilenberg-Mac Lane mapping algebra, these higher order Ext-groups compute the $E_{m}$-terms of the Adams spectral sequence for $m \leq n+2$.

See Theorems 15.9 and 15.11 below.

0.2. Remark. As shown in [BJ2], at the secondary level (that is, computing the $d_{2^{-}}$ differential), this algebra of cubical balls can be replaced by an ordinary differential graded algebra. In $[\mathrm{BaF}]$, the first author and Martin Frankland show that this can also be done at the tertiary level. It is conjectured in [B3] that there is a single differential graded algebra, extracted from the algebra of cubical balls, from which the whole Adams spectral sequence can be computed. 
0.3. Remark. The associative composition between mapping spaces induces an associative operation $\otimes$ on singular cubes, because the product of cubes is again a cube. This property does not hold for simplices, which is why we chose such singular cubes as our main technical tool here. In $[\mathrm{BaF}]$, too, the $\otimes$-product of cubes plays a central role. This is because the geometry of cubes is better suited to describing composition of (higher) homotopies than the geometry of simplices.

We observe that, in the same way, cubes (rather than simplices) are used in the literature to describe higher order homotopy operations, such as Toda brackets (see $[\mathrm{BJT}]$ ) and the higher homotopy commutativity of diagrams (see [BV]).

0.4. Remark. Note that the Steenrod squares, represented by Steenrod's original $\cup_{i^{-}}$ operations at the chain level, generate the whole Steenrod algebra $\mathcal{A}$. In [K1, K2], Kristensen used these $\cup_{i}$-operations to calculate secondary cohomology operations. Similarly, the algebra of left cubical balls associated to the Eilenberg-Mac Lane mapping algebra can be used to extend Kristensen's approach to higher dimensions.

0.5. Remark. This paper is framed in terms of the Eilenberg-Mac Lane mapping algebra, since the most important application of our methods is to the classical Adams spectral sequence. However, our methods may be extended to other mapping algebras derived from other (topologically-enriched) Quillen model categories.

0.6. Convention. The category of compactly generated Hausdorff spaces (cf. [S], and compare $[\mathrm{V}]$ ) is denoted by Top, and that of pointed connected compactly generated spaces by $\mathbf{T o p}_{*}$. In particular, we shall assume that all (pointed) topological mapping spaces in this paper take value in $(\mathbf{T o p}, \times) \quad\left(\right.$ respectively, $\left.\left(\mathbf{T o p}_{*}, \wedge\right)\right)$. See also $[\mathbf{W h}$, $\S 1.1]$.

0.7. Acknowledgements. The authors wish to thank the referee for his or her careful reading of the paper and many pertinent remarks. The first author would like to thank the Department of Mathematics at the University of Haifa, and the second author would like to thank the Max Planck Institut für Mathematik in Bonn, for their hospitality while this research was being carried out. The second author was partly supported by Israel Science Foundation Grant 247377/11.

\section{LEFT CUBICAL SETS}

A nullhomotopy is a homotopy between some map and the zero map between pointed topological spaces. We wish to consider higher homotopies, which are described by singular cubes in mapping spaces; in particular, we have higher nullhomotopies, which lead us to the concept of left cubical sets, defined in this section. The main examples we shall need are the left $n$-cubical sets $\operatorname{nul}_{n}(X)$ and $\operatorname{Nul}_{n}(X)$ of a pointed space $X$.

We first recall some basic properties of cubical sets: let $I=[0,1]$ be the unit interval and let $I^{n}=I \times \cdots \times I$ be the $n$-dimensional cube. We have inclusions $d_{\varepsilon}^{i}: I^{n-1}=I^{i-1} \times\{\varepsilon\} \times I^{n-i} \subset I^{n}$ for $1 \leq i \leq n$ and $\varepsilon \in\{0,1\}$. Here $I^{0}$ is a single point.

Let $\square$ denote the category whose objects are cubes $I^{n} \quad(n \geq 0)$, and whose morphisms are generated by $d_{\varepsilon}^{i}$ and the projections $s^{i}: I^{n} \rightarrow I^{n-1}$.

A pointed cubical set is a functor $K: \square^{\mathrm{op}} \longrightarrow \mathbf{S e t}_{*}$, where $\mathbf{S e t}_{*}$ is the category of pointed sets. As usual, $K\left(I^{n}\right)$ is denoted $K_{n}$ and $* \in K_{n}$ is the base point. We 
write $\operatorname{dim}(a)=n$ if $a \in K_{n}$. See [C], [J], or [I] for further details on the category of cubical sets.

1.1. Definition. Let $\square$ be the subcategory of $\square$ consisting of objects $I^{n} \quad(n \geq 0)$ and morphisms generated by $d_{0}^{i}$. A left cubical set is a functor $\bar{\square}^{\text {op }} \rightarrow$ Set $_{*}$. We write $\partial^{i} \quad$ for $\left(d_{0}^{i}\right)^{*}: K_{n} \rightarrow K_{n-1} \quad(1 \leq i \leq n)$. We also consider the full subcategories $\bar{\square}_{n} \subset \bar{\square}$ consisting of objects $I^{m} \quad(0 \leq m \leq n)$. A functor $\bar{\square}_{n}^{\mathrm{op}} \longrightarrow$ Set $_{*}$ is called a left $n$-cubical set.

1.2. Example. Given a pointed cubical set $K$, one obtains a left cubical set $\operatorname{nul}(K)$ by setting

$$
\operatorname{nul}(K)_{m}:=\left\{a \in K_{m} \mid \quad\left(d_{1}^{i}\right)^{*} a=* \text { for } 1 \leq i \leq m\right\} .
$$

Accordingly, we define the left $n$-cubical set $\operatorname{nul}_{n}(K)$ to be the restriction of $\operatorname{nul}(K)$ to $\bar{\square}_{n}^{\text {op }}$.

1.3. Remark. The following properties of (left) cubical sets are included here for future reference. Observe that nul is a functor from pointed cubical sets to left cubical sets. Its left adjoint $\mathcal{U}: \mathbf{S e t}_{*}^{\square^{\mathrm{op}}} \rightarrow \mathbf{S e t}_{*}^{\square^{\text {op }}}$ may be thought of as a "universal enveloping cubical set" functor, described as follows: given a left cubical set $M$, the pointed cubical set $\mathcal{U}(M)$ has one $n$-cube $I_{a}^{n}$ for each left $n$-cube $a \in M$, with $\left(d_{1}^{i}\right)^{*} I_{a}^{n}=* \quad$ (the base point) for each $1 \leq i \leq n$.

In addition, there is a degenerate $(n+k)$-cube:

$\left(s^{j_{1}}\right)^{*} \ldots\left(s^{j_{k}}\right)^{*} I_{a}^{n}$ in $\mathcal{U}(M)$ for each iterated projection $s^{j_{k}} \ldots s^{j_{1}}: I^{n+k} \rightarrow I^{n}$ in

(with identifications according to the cubical identities).

It is readily verified that $\mathcal{U}(M)$ is indeed a pointed cubical set, with a natural isomorphism:

$$
\operatorname{Hom}_{\mathbf{S e t}_{*}^{\square^{\mathrm{op}}}}(M, \operatorname{nul}(K)) \stackrel{\cong}{\rightarrow} \operatorname{Hom}_{\mathbf{S e t}_{*}^{\square o p}}(\mathcal{U}(M), K)
$$

for $K \in \mathbf{S e t}_{*}^{\square^{\mathrm{op}}}$ and $M \in \mathbf{S e t}_{*}^{\square^{\mathrm{op}}}$. Moreover, both functors preserve dimensions of all cubes, so they commute with the $n$-skeleton functor, yielding a left adjoint $\mathcal{U}_{n}$ to $\mathrm{nul}_{n}$.

For any cubical set $K$, let $\mathcal{C}_{K}$ be the partially ordered set of all $k$-cubes $(k \geq 0)$ of $K$, ordered under inclusion. We have $K \cong \operatorname{colim}_{I^{k} \in \mathcal{C}_{K}} I^{k}$, where each $I^{k}$ is thought of as a cubical set. We use this to define a monoidal structure on Set $^{\square^{o p}}$, given by:

$$
K \otimes L:=\operatorname{colim}_{I^{j} \in \mathcal{C}_{K}, I^{k} \in \mathcal{C}_{L}} I^{j+k}
$$

(see [J, §3]). If $K$ and $L$ are pointed, there is a cubical smash functor

$$
K \bar{\otimes} L:=(K \otimes L) /(\{*\} \otimes L \amalg K \otimes\{*\})
$$

on $\mathbf{S e t}_{*}^{\square^{\text {op }}}$, which is also defined on $\mathbf{S e t}_{*}^{\bar{\square}^{\text {op }}}$. Moreover, nul and $\mathcal{U}$ are monoidal with respect to $\bar{\otimes}$ on $\mathbf{S e t}_{*}^{\square^{\mathrm{op}}}$ and $\mathbf{S e t}_{*}^{\bar{D}^{\mathrm{op}}}$, respectively.

1.8. Definition. For a pointed space $(X, *)$, let $\mathcal{S}_{\square} X$ be the singular pointed cubical set: thus $\left(\mathcal{S}_{\square} X\right)_{n}$ is the set of all maps $I^{n} \longrightarrow X$, with base point $o: I^{n} \longrightarrow\{*\} \subset X$. 
Thus, the set $\operatorname{nul}(X):=\operatorname{nul}\left(\mathcal{S}_{\square} X\right)$ of all singular null cubes in $X$ consists of all maps $a: I^{n} \rightarrow X \quad(n \geq 0)$ with $a d_{1}^{i}=o$ for $1 \leq i \leq n$. We let $\operatorname{nul}_{n}(X):=\operatorname{nul}_{n}\left(\mathcal{S}_{\square} X\right)$.

1.9. Definition. For any pointed topological space $(X, *)$, the left $n$-cubical set $\mathrm{Nul}_{n}(X)$ is defined by

$$
\operatorname{Nul}_{n}(X)_{m}:=\left\{\begin{array}{l}
\operatorname{nul}(X)_{m} \text { for } m<n, \\
\operatorname{nul}(X)_{n} / \simeq \text { for } n=m .
\end{array}\right.
$$

Here we set $a \simeq b$ for $a, b \in \operatorname{nul}(X)_{n}$ if the maps $a, b: I^{n} \longrightarrow X$ are homotopic relative to the boundary $\partial I^{n}$ of the cube $I^{n}$. Let $\{a\}$ be the equivalence class of $a$; we call $\{a\}$ an $n$-track in $X$.

There is a surjective map of left $n$-cubical sets:

$$
\operatorname{nul}_{n}(X) \rightarrow \operatorname{Nul}_{n}(X),
$$

which is the identity in dimension $<n$ and which carries $a$ with $\operatorname{dim}(a)=n$ to the $n$-track $\{a\}$. We point out that the left $n$-cubical set $\operatorname{Nul}_{n}(X)$ is not the restriction of a cubical set.

1.11. Remark. Let $\bar{\triangle}$ be the category with sets $\{1,2, \ldots, n\} \quad(n \geq 0)$ as objects, and order preserving injective maps as morphisms. There is an isomorphism of categories $\bar{\triangle} \cong \bar{\square}$ which carries $\{1,2, \ldots, n\}$ to $I^{n}$ and carries

$$
\{1,2, \ldots, n-1\} \cong\{1, \ldots, \widehat{i}, \ldots, n\} \subset\{1, \ldots, n\}
$$

to $d_{0}^{i}$. Here $\widehat{i}$ indicates that we omit $i$.

\section{2. $n$-GRADED CATEGORIES ENRICHED IN LEFT CUBICAL SETS}

In this section we define the $\otimes$-composition of singular cubes in a topologically enriched category, using the fact that the product of two cubes is a cube. It is important to note that (in a category enriched in $\left(\mathbf{T o p}_{*}, \wedge\right)$ - see 80.6 ) the $\otimes$-composition of two singular null cubes is a singular null cube. The $\otimes$-composition respects the obvious grading by dimension, yielding a graded category:

2.1. Definition. A graded set is a sequence of sets $K=\left(K_{n}\right)_{n=0}^{\infty}$, and a map of graded sets $f: K \rightarrow L$ is a sequence of maps $f_{n}: K_{n} \rightarrow L_{n} \quad(n \geq 0)$. We write $\operatorname{dim}(x)=n$ if $x \in K_{n}$. A graded category $\mathbf{G}$ is a category in which each morphism $f$ has a dimension $\operatorname{dim}(f) \geq 0$ such that the composition $f g$ satisfies

$$
\operatorname{dim}(f g)=\operatorname{dim}(f)+\operatorname{dim}(g) .
$$

Thus, all morphism sets $\operatorname{Mor}_{\mathbf{G}}(X, Y)$ are graded sets.

An $n$-set $L$ is a finite sequence of sets $\left(L_{0}, \ldots, L_{n}\right)$. For example, the $n$-skeleton $\left(K_{0}, \ldots, K_{n}\right)$ of a graded set is an $n$-set. An $n$-graded category consists of morphism sets which are $n$-sets and composition $f g$ is defined if $\operatorname{dim}(f)+\operatorname{dim}(g) \leq n$. For example, the $n$-skeleton of a graded category is an $n$-graded category. 
An $n$-graded category enriched in left cubical sets is an $n$-graded category such that morphism sets $\operatorname{Mor}(X, Y)$ are left $n$-cubical sets with operators $\left(d_{0}^{i}\right)^{*}=\partial^{i}$ satisfying

$$
\partial^{i}(f g)= \begin{cases}\left(\partial^{i} f\right) g & \text { for } i \leq \operatorname{dim}(f) \\ f\left(\partial^{i-\operatorname{dim}(f)} g\right) & \text { for } i>\operatorname{dim}(f)\end{cases}
$$

Moreover, the zero maps $o^{n} \in \operatorname{Mor}(X, Y)_{n} \quad(n \geq 0)$ satisfy

$$
o^{n} g=o^{n+\operatorname{dim}(g)} \text { and } f o^{m}=o^{\operatorname{dim}(f)+m} .
$$

2.3. Example. Let $\mathbf{C}$ be a category enriched in $\left(\mathbf{T o p}_{*}, \wedge\right)$ where $\wedge$ is the smash product of pointed topological spaces (cf. \$0.6). Thus for every $X, Y \in \operatorname{Obj}(\mathbf{C})$, there is a zero map $o \in \operatorname{Mor}_{\mathbf{C}}(X, Y)$, satisfying $o g=o$ and $f_{o}=o$ for any $f, g \in \operatorname{Mor}_{\mathbf{C}}$.

The $\otimes$-composition of two singular (null) cubes $f \otimes g$ is defined to be the composite

$$
f \otimes g: I^{n} \times I^{m} \stackrel{f \times g}{\longrightarrow} \operatorname{Mor}_{\mathbf{C}}(Y, X) \times \operatorname{Mor}_{\mathbf{C}}(Z, Y) \stackrel{\mu}{\longrightarrow} \operatorname{Mor}_{\mathbf{C}}(Z, X),
$$

where $\mu$ is the composition in $\mathbf{C}$. We see that the $\otimes$-composition of two singular null cubes is a singular null cube, so the category nul $\mathbf{C}$ is a enriched in left cubical sets, with nul $\mathbf{C}(X, Y):=\operatorname{nul} \operatorname{Mor}_{\mathbf{C}}(X, Y)$.

2.4. Definition. For $\mathbf{C}$ as above, the $n$-skeleton of nul $\mathbf{C}$, denoted by $\operatorname{nul}_{n} \mathbf{C}$, is given by the $n$-cubical sets $\operatorname{nul}_{n} \operatorname{Mor}_{\mathbf{C}}(X, Y)$, and $\operatorname{Nul}_{n} \mathbf{C}$ is defined similarly. There is a quotient functor

$$
\operatorname{nul}_{n} \mathbf{C} \rightarrow \mathrm{Nul}_{n} \mathbf{C}
$$

given by the quotient maps:

$$
\operatorname{nul}_{n} \operatorname{Mor}_{\mathbf{C}}(X, Y) \longrightarrow \operatorname{Nul}_{n} \operatorname{Mor}_{\mathbf{C}}(X, Y)
$$

(see $(1.10)$ ).

Thus $\mathrm{Nul}_{n} \mathbf{C}$ is an $n$-graded category with the composition defined by the equivalence class $\{f \otimes g\}$ for $\operatorname{dim}(f)+\operatorname{dim}(g)=n$. The $n$-graded categories $\operatorname{nul}_{n} \mathbf{C}$ and $\mathrm{Nul}_{n} \mathbf{C}$ are enriched in left $n$-cubical sets.

These two examples $\operatorname{nul}_{n} \mathbf{C}$ and $\mathrm{Nul}_{n} \mathbf{C}$ are the reason for our definition of the partially-defined composition in $n$-graded categories in $\$ 2.1$ above.

We also consider functors between $n$-graded categories, and in the next section we will use such functors to define higher order chain complexes.

For $n=0$, the (0-graded) category

$$
\mathrm{Nul}_{0} \mathbf{C}=\pi_{0} \mathbf{C}
$$

has morphisms $X \longrightarrow Y$ given by the path components of $\operatorname{Mor}_{\mathbf{C}}(X, Y)$.

\section{The Chain CATEgory $\mathbb{Z}_{\otimes}$}

A chain complex in any pointed category $\mathcal{M}$ may be defined as a pointed functor from a suitable indexing category. A higher order chain complex is a functor between $n$-graded categories, from a more elaborate indexing category $\mathbb{Z}_{\otimes}^{n} \quad$ (which we now describe) into the category $\mathrm{Nul}_{n} \mathbf{C}$ of 92.4 . The reader can skip the explicit definitions of $\mathbb{Z}_{\otimes}$ and $\mathbb{Z}_{\otimes}^{n}$ in $\$ 3.1$ and $\$ 3.3$, we only need to know that they are well-defined. 
3.1. Definition. Let $N:=\operatorname{Mon}(\star, J)$ be the free monoid generated by elements $\star$ and $J$, so the elements of $N$ are words in the letters $\star$ and $J$.

Let $\operatorname{deg}, \operatorname{dim}: N \longrightarrow\left(\mathbb{N}_{0},+\right)$ be monoid homomorphisms defined by

$$
\begin{aligned}
& \operatorname{deg}(\star)=1 \\
& \operatorname{deg}(J)=1 \\
& \operatorname{dim}(\star)=0 \\
& \operatorname{dim}(J)=1 .
\end{aligned}
$$

For example, $V=\star \star J \star J J$ is a word in $N$, with $\operatorname{deg}(V)=6$ the length of the word $V$, and $\operatorname{dim}(V)=3$ the number of letters $J$ in $V$. Let $\emptyset$ be the empty word, which is the unit in the monoid $N$.

We associate with $J$ the unit interval $I=[0,1]$ and with $\star$ the one point space $\{0\}$. For any word $V$, let $\bar{V}$ be the space defined by

$$
\bar{V}= \begin{cases}I & \text { if } V=J \\ \{0\} & \text { if } V=\star \\ \bar{V}_{1} \times \bar{V}_{2} & \text { if } V=V_{1} V_{2} .\end{cases}
$$

We say that $V$ is in the boundary of $W$ with $V, W \in N \quad(V \neq W)$ if there is an inclusion $\bar{V} \subset \bar{W}$. This implies $\operatorname{deg} V=\operatorname{deg} W$ and $\operatorname{dim} V \leq \operatorname{dim} W . \quad$ By projecting the spaces $\{0\}$, one gets the homeomorphism

$$
\bar{V} \cong I^{\operatorname{dim} V} .
$$

If $V$ is in the boundary of $W$, there is a unique inclusion $d_{V, W}$ of cubes in the category $\bar{\square}$ (see Definition 1.1 ) such that

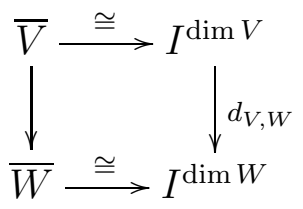

commutes.

Now, consider elements $\star$ and $I_{n} \quad(n \geq 1)$, which generate the monoid

$$
M:=\operatorname{Mon}\left(\star, I_{n}: n \geq 1\right) / I_{n} \circ I_{m}=I_{n+m} .
$$

The multiplication in $M$ is denoted by $\circ$. Here, $\operatorname{Mon}\left(\star, I_{n}: n \geq 1\right)$ denotes the free monoid. In $M$, we divide out by the relation $I_{n} \circ I_{m}=I_{n+m}$ for $n, m \geq 1$.

There is a canonical isomorphism of monoids

$$
M \stackrel{\cong}{\longrightarrow} N
$$

which carries $\star$ to $\star$ and $I_{n}$ to the $n$-fold product $J^{n}=J \cdots J$. Using this isomorphism, we obtain the functions $\operatorname{deg}$ and $\operatorname{dim}$ on $M$.

We introduce on $M$ a further multiplication $\otimes$ defined by

$$
V \otimes W=V \circ \star \circ W \quad \text { for } V, W \in M,
$$

where the right hand side is the product of the elements $V$, $\star$, and $W$ in the monoid $M$. The operation $\otimes$ is associative, but it has no unit. For the empty word $\emptyset \in M$, we get

$$
\emptyset \otimes \emptyset=\emptyset \circ \star \circ \emptyset=\star
$$


3.3. Definition. We define the chain category $\mathbb{Z}_{\otimes}$ to be the following graded category: the objects in $\mathbb{Z}_{\otimes}$ are the integers $i, j, \ldots \in \mathbb{Z}$. In addition to the identities $1_{i}$, with $\operatorname{dim}\left(1_{i}\right)=0$, the morphisms in $\mathbb{Z}_{\otimes}$ consist of

$$
(i, V): i \stackrel{V}{\longrightarrow} i-\operatorname{deg} V-1=j
$$

for all $V \in M$. The composition of $V: i \longrightarrow j$ and $W: j \longrightarrow j-\operatorname{deg} W-1=k$ $(W \in M)$, is defined

$$
(i, W \otimes V): i \stackrel{W \otimes V}{\longrightarrow} i-\operatorname{deg}(W \otimes V)-1=k .
$$

Here we have $\operatorname{deg}(W \otimes V)=\operatorname{deg} W+\operatorname{deg} V+1$, so that the composition is well defined. We also omit $\otimes$ in the notation of the composite.

More precisely, morphisms in $\mathbb{Z}_{\otimes}$ are pairs $(i, V)$, where $i \in \mathbb{Z}, \quad V \in M$, and $i$ is the source of the morphism $(i, V)$ (also written $V: i \longrightarrow j$ ). The target $j$ satisfies $j=i-\operatorname{deg} V-1$.

See Section 5 below for an alternative point of view.

The category $\mathbb{Z}_{\otimes}$ is graded by dimension of elements in $M$, with $\operatorname{dim}(W \otimes V)=$ $\operatorname{dim}(W)+\operatorname{dim}(V)$. The $n$-skeleton $\mathbb{Z}_{\otimes}^{n}$ of $\mathbb{Z}_{\otimes} \quad(n \geq 0)$ is an $n$-graded category. The 0 -skeleton $\mathbb{Z}_{\otimes}^{0}$ consists only of identities and of the morphisms $V: i \rightarrow i-\operatorname{deg} V-1$, where $V$ is a power of the element $\star$ in $M$.

If $V=\emptyset$, then $\emptyset: i \longrightarrow i-1$ is in $\mathbb{Z}_{\otimes}^{0}$. The composition is $\emptyset \otimes \emptyset=\star: i \longrightarrow i-2$, and so on. We observe:

3.4. Lemma. The category $\mathbb{Z}_{\otimes}$ is freely generated by the morphisms $(i, \emptyset): i \rightarrow i-1$ and $\left(i, I_{k}\right): i \longrightarrow i-k-1$ for $i \in \mathbb{Z} . \quad k \geq 1$.

\section{Higher ORDER CHAin COMPLEXES}

We are now in a position to define higher order chain complexes. In Section 8 , we will introduce the notion of a higher order resolution, which is a special kind of higher order chain complex.

4.1. Definition. Given an $n$-graded category $\mathbf{T}$ enriched in left $n$-cubical sets (such as $\mathbf{T}=\mathrm{Nul}_{n} \mathbf{C}$ ), we consider a functor of $n$-graded categories

$$
K: \mathbb{Z}_{\otimes}^{n} \rightarrow \mathbf{T}
$$

which carries an object $i \in \mathbb{Z}$ to the object $K_{i}:=K(i) \quad$ in $\mathbf{T}$. We say that $K$ satisfies the inclusion property if the following holds:

Given morphisms $V, W: i \longrightarrow j$ in $\mathbb{Z}_{\otimes}^{n}$ such that $V$ is in the boundary of $W$, then the induced morphisms $K(V)$ and $K(W)$ in $\mathbf{T}$ satisfy the equation

$$
K(V)=d_{V, W}^{*} K(W) \quad \text { in } \operatorname{Mor}_{\mathbf{T}}\left(K_{i}, K_{j}\right) .
$$

Here $d_{V, W}^{*}$ is defined by the structure of $\operatorname{Mor}_{\mathbf{T}}\left(K_{i}, K_{j}\right)$ as a left cubical set.

A functor $K$ satisfying the inclusion property (4.2) is called an $n$-th order pre-chain complex in $\mathbf{T}$.

4.3. Definition. Let $N>M$ and $\mathbb{Z}(N, M)=\{k \in \mathbb{Z}, N \geq k \geq M\}$. Then we obtain the full subcategory

$$
\mathbb{Z}(N, M)_{\otimes} \subset \mathbb{Z}_{\otimes}
$$


consisting of objects $k \in \mathbb{Z}(N, M)$. We say that $K$ is concentrated in $\mathbb{Z}(N, M)$ if $K: \mathbb{Z}(N, M)_{\otimes}^{n} \longrightarrow \mathbf{T} \quad$ is a functor of $n$-graded categories.

Assume given a quotient functor $\mathbf{T}^{0} \longrightarrow \mathbf{A}$, (that is, a full functor which is the identity on objects) yielding induced morphisms

$$
\delta_{i}=K(i, \emptyset)_{*}: K_{i} \rightarrow K_{i-1}
$$

in $\mathbf{A}$ for each $i \in \mathbb{Z}$. We then say that $K$ is based on the diagram

$$
K_{M} \leftarrow \ldots \leftarrow K_{i-2} \stackrel{\delta_{i-1}}{\longleftarrow} K_{i-1} \stackrel{\delta_{i}}{\leftarrow} K_{i} \leftarrow \ldots \leftarrow K_{N}
$$

in the category $\mathbf{A}$.

4.5. Definition. Let $\mathbf{C}$ be a category enriched in pointed spaces (with zero maps $o$ ). For $\mathbf{T}=\mathrm{Nul}_{n} \mathbf{C}$, we consider a functor $K$ with the inclusion property,

$$
K: \mathbb{Z}_{\otimes}^{n} \longrightarrow \mathrm{Nul}_{n} \mathbf{C} \text {. }
$$

We have in $\mathbb{Z}_{\otimes}^{n}$ the $(n+1)$-tuple of morphisms $i \longrightarrow i-n-2$ :

$$
\left(i, \partial I_{n+1}\right)=\left\{\begin{array}{l}
\left(i, \emptyset \otimes I_{n}\right), \\
\left(i, I_{n} \otimes \emptyset\right), \\
\left(i, I_{r} \otimes I_{s}\right), \quad r+s=n, r \geq 1, s \geq 1
\end{array}\right.
$$

which yields the $(n+1)$-tuple of $n$-tracks

$$
K\left(i, \partial I_{n+1}\right)=\left(K\left(i, \emptyset \otimes I_{n}\right), K\left(i, I_{1} \otimes I_{n-1}\right), \ldots, K\left(i, I_{n-1} \otimes I_{1}\right), K\left(i, I_{n} \otimes \emptyset\right)\right) .
$$

These tracks are represented by maps $I^{n} \longrightarrow \operatorname{Mor}_{\mathbf{C}}\left(K_{i}, K_{i-n-2}\right)$. In fact, these $n$-tracks yield a map

$$
\alpha: S^{n} \approx \partial\left(I^{n+1}\right) \longrightarrow \operatorname{Mor}_{\mathbf{C}}\left(K_{i}, K_{i-n-2}\right)
$$

on the boundary of the $(n+1)$-cube, well-defined up to homotopy. Hence, the map $\alpha$ determines an obstruction element

$$
\mathcal{O} K\left(i, \partial I_{n+1}\right) \in \pi_{n} \operatorname{Mor}_{\mathbf{C}}\left(K_{i}, K_{i-n-2}\right) .
$$

We write $D_{n}\left(K_{i}, K_{i-n-2}\right)$ for $\pi_{n} \operatorname{Mor}_{\mathbf{C}}\left(K_{i}, K_{i-n-2}\right)$, since these form a natural system $D_{n}$ (see $\$ 7.1$ below).

We say that $K$ is an $n$-th order chain complex in $\mathrm{Nul}_{n} \mathbf{C}$ if the obstruction elements (4.7) vanish for all $i$. This is the obstruction property of $K$.

In Section 11 below, we determine the basic formulas satisfied by these obstruction elements.

4.8. Definition. For $\mathbf{C}$ as above, let

$$
K_{0} \stackrel{\delta_{1}}{\longleftarrow} K_{1} \stackrel{\delta_{2}}{\longleftarrow} K_{2} \longleftarrow \ldots \stackrel{\delta_{n+2}}{\longleftarrow} K_{n+2}
$$

be a diagram in $\mathbf{A}=\pi_{0}(\mathbf{C}) \quad(n \geq 1)$. Consider all functors

$$
K: \mathbb{Z}(0, n+2)_{\otimes}^{n} \rightarrow \mathbf{T}=\mathrm{Nul}_{n} \mathbf{C}
$$

based on the diagram (4.9) which satisfy the inclusion property. Each such functor yields an obstruction element

$$
\mathcal{O} K\left(n+2, \partial I_{n+1}\right) \in D_{n}\left(K_{n+2}, K_{0}\right) .
$$


The set of all these elements is the classical higher order Toda bracket

$$
\left\langle\delta_{1}, \ldots, \delta_{n+2}\right\rangle \subset D_{n}\left(K_{n+2}, K_{0}\right)
$$

(see [Wa]).

The set can be empty. If there exists an $n$-th order chain complex $K$ based on diagram (4.9), then of course $0 \in\left\langle\delta_{1}, \ldots, \delta_{n+2}\right\rangle$, by the obstruction property of $K$.

\section{The $W$-COnstruction}

We now present an alternative description of higher order chain complexes not actually needed for this paper, based on the classical bar construction $W \mathcal{K}$ going back to Boardman-Vogt (see [BV, §3] and [Bo, §6]). This construction is a topologicallyenriched "cofibrant replacement" for any small category $\mathcal{K}$ (not necessarily unital), which serves as the indexing category for lax versions of functors $\mathcal{K} \rightarrow$ Top. A cubically enriched variant of $W \mathcal{K}$ was defined in [BJT, §3.1] and [BB1, §3.4]; we shall require the following pointed setting:

5.1. Definition. Let $\mathcal{K}$ be a small category enriched in $\left(\right.$ Set $_{*}, \wedge$ ) (with zero maps $o$ ). The pointed $W$-construction on $\mathcal{K}$, denoted by $W_{*} \mathcal{K}$, is the (non-unital) category enriched in $\left(\mathbf{S e t}_{*}^{\square^{\circ p}}, \bar{\otimes}\right)$ with object set $\operatorname{Obj} \mathcal{K}$ defined as follows:

First, for every $a, b \in \operatorname{Obj} \mathcal{K}$, the underlying graded pointed category of $W_{*} \mathcal{K}$ has an (indecomposable) morphism $\left(n\right.$-cube) $I_{f_{\bullet}}^{n}$ in $W_{*} \mathcal{K}(a, b)_{n}$ associated to each composable sequence

$$
f_{\bullet}=\left(a=a_{n+1} \stackrel{f_{n+1}}{\longrightarrow} a_{n} \stackrel{f_{n}}{\longrightarrow} a_{n-1} \ldots a_{1} \stackrel{f_{1}}{\longrightarrow} a_{0}=b\right)
$$

of length $n+1$ in $\mathcal{K}$. In addition, $W_{*} \mathcal{K}(a, b)$ has a degenerate $(n+k)$-cube $\left(s^{j_{1}}\right)^{*} \ldots\left(s^{j_{k}}\right)^{*} I_{f_{\bullet}}^{n} \quad$ for each iterated projection $s^{j_{k}} \ldots s^{j_{1}}: I^{n+k} \rightarrow I^{n} \quad$ in $\square$ (with identifications according to the cubical identities). The zero map in degree $k$ is $I_{o}^{k}:=$ $\left(s_{k}\right)^{*} \ldots\left(s_{1}\right)^{*} I_{o}^{0}$, and we identify $I_{f_{\bullet}}^{n}$ with $I_{o}^{n}$ whenever at least one of the maps $f_{1}, \ldots, f_{n+1}$ is $o$. Then $W_{*} \mathcal{K}$ is freely generated as a graded category with zero maps by these cubes. Composition in the category $W_{*} \mathcal{K}$ is denoted by $\bar{\otimes}$.

The cubical structure is determined by the face maps of the non-degenerate indecomposable cubes $I_{f_{\bullet}}^{n}$ and the cubical identities, as follows:

(a) The $i$-th 1 -face of $I_{f_{\bullet}}^{n} \quad$ is $I_{f_{1} \circ \ldots \circ\left(f_{i} \cdot f_{i+1}\right) \circ \ldots f_{n+1}}^{n-1}$ - that is, we carry out (in the category $\mathcal{K}$ ) the $i$-th composition in $f_{0}$.

(b) The $i$-th 0 -face of $I_{f_{\bullet}}^{n}$ is the composite $I_{f_{1} \circ \ldots \circ f_{i}}^{i-1} \bar{\otimes} I_{f_{i+1} \circ \ldots \circ f_{n+1}}^{n-i}$.

(c) The cubical structure on the composites $I_{f_{\bullet}}^{j} \bar{\otimes} I_{g_{\bullet}}^{k}$ is defined by (1.6) (or $(2.2)$ ).

5.3. Definition. Let $\Gamma$ be the category enriched in $\left(\mathbf{S e t}_{*}, \wedge\right)$ with object set $\mathbb{Z}$ and a single non-zero arrow $d_{k+1}: k+1 \rightarrow k$ for each $k \in \mathbb{Z}$, satisfying $d_{k} \circ d_{k+1}=o$ for all $k$.

5.4. Proposition. Let $\mathcal{M}$ be a category enriched in cubical sets with zero maps. There is a one-to-one correspondence between pointed cubical functors $W_{*} \Gamma \rightarrow \mathcal{M}$ and pre-chain complexes in nul $\mathcal{M}$, which restricts to a one-to-one correspondence between pointed cubical functors $\operatorname{sk}_{n} W_{*} \Gamma \rightarrow \mathcal{M}$ and $n$-th order pre-chain complexes in $\operatorname{nul}_{n} \mathcal{M}$. 
Proof. Since $\mathbb{Z}_{\otimes}$ is a free graded category, by Lemma 3.4, we can define a one-to-one functor of graded categories $\Phi: \mathbb{Z}_{\otimes} \rightarrow W_{*} \Gamma$ which is the identity on objects by setting $\Phi(i, \emptyset):=I_{d_{i}}^{0}$ and $\Phi\left(i, I_{k}\right):=I_{f_{\bullet}}^{k}$, for $f_{\bullet}:=\left(i \stackrel{d_{i}}{\rightarrow} i-1 \rightarrow \ldots i-k \stackrel{d_{i-k}}{\longrightarrow} i-k-1\right)$.

We can think of $\mathbb{Z}_{\otimes}$ as a category $\widehat{\mathbb{Z}}_{\otimes}$ enriched in $\left(\right.$ Set $\left._{*}^{\bar{D}^{\mathrm{op}}}, \bar{\otimes}\right)$ by setting $d_{V, W}^{*}(W)=V$ if $V \subseteq W$, and adding zero maps. Note that a functor $K: \mathbb{Z}_{\otimes} \rightarrow$ nul $\mathcal{M}$ is a pre-chain complex if and only if it induces a pointed cubical functor $\hat{K}: \widehat{\mathbb{Z}}_{\otimes} \rightarrow \operatorname{nul} \mathcal{M}$.

The universal enveloping functor $\mathcal{U}: \mathbf{S e t}_{*}^{\bar{\square}^{\mathrm{op}}} \rightarrow \mathbf{S e t}_{*}^{\square^{\text {op }}}$ of Remark 1.3 is monoidal with respect to $\bar{\otimes}$, so the adjunction (1.5) extends to categories of enriched functors. Moreover, $\Phi$ induces a natural isomorphism of pointed cubical categories

$$
\mathcal{U}\left(\widehat{\mathbb{Z}}_{\otimes}\right) \cong W_{*} \Gamma
$$

so left cubical functors $\widehat{\mathbb{Z}}_{\otimes} \rightarrow$ nul $\mathcal{M}$ indeed correspond to pointed cubical functors $W_{*} \Gamma \rightarrow \mathcal{M}$. Since this correspondence preserves the grading, the same is true for $n$-th order pre-chain complexes.

\section{RESOLUTIONS AND DERIVED FUNCTORS}

We now recall the classical definitions of derived functors and resolutions (which are ordinary chain complexes) in additive categories. Our higher order resolutions will be higher order chain complexes based on such ordinary resolutions (in the sense of 94.3 ).

6.1. Definition. Let $\mathbf{A}$ be a category enriched in abelian groups, i.e., a preadditive category. We denote the morphism sets for objects $X, Y$ in $\mathbf{A}$ by

$$
\operatorname{Hom}_{\mathbf{A}}(X, Y)=\operatorname{Mor}_{\mathbf{A}}(X, Y) \text {. }
$$

This is an abelian group, and morphisms $f: X^{\prime} \longrightarrow X$ and $g: Y^{\prime} \longrightarrow Y$ in $\mathbf{A}$ induce homomorphisms $\operatorname{Hom}(f, Y)$ and $\operatorname{Hom}(X, g)$. Let a be a full subcategory of A.

Let $Y$ be an object in A. An a-resolution of $Y$ is a diagram

$$
A_{\bullet}=\left(\ldots \stackrel{\delta_{2}}{\longrightarrow} A_{1} \stackrel{\delta_{1}}{\longrightarrow} A_{0} \stackrel{\delta_{0}}{\longrightarrow} A_{-1}\right)
$$

in $\mathbf{A}$ with $A_{-1}=Y$ and $A_{i} \in \mathbf{a}$ for $i \geq 0$, such that, for all objects $B$ in a, the induced diagram $\operatorname{Hom}\left(B, A_{\bullet}\right)$ is an exact sequence of abelian groups; in particular, $\operatorname{Hom}\left(B, \delta_{0}\right)$ is surjective.

An a-coresolution of $X$ is a diagram

$$
A^{\bullet}=\left(A_{1} \stackrel{\delta_{1}}{\longrightarrow} A_{0} \stackrel{\delta_{0}}{\longrightarrow} A_{-1} \stackrel{\delta_{-1}}{\longrightarrow} \ldots\right)
$$

in A with $A_{1}=X$ and $A_{i} \in \mathbf{a}$ for $i \geq 0$, such that for all objects $B$ in a the induced diagram $\operatorname{Hom}\left(A^{\bullet}, B\right)$ is an exact sequence of abelian groups. Here $\operatorname{Hom}\left(\delta_{1}, B\right)$ is surjective.

The next result is proved in [BJ2, 1.3]:

\subsection{Lemma. Suppose}

(1) the coproduct of any family of objects of $\mathbf{a}$ exists in $\mathbf{A}$ and belongs to a again,

(2) there is a small subcategory $\mathbf{g}$ of $\mathbf{a}$ such that every object of $\mathbf{a}$ is a retract of a coproduct of a family of objects from $\mathbf{g}$, 


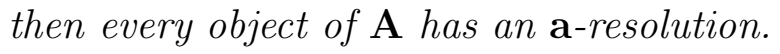

The dual statement also holds:

\subsection{Lemma. Suppose}

(1)' the product of any family of objects of $\mathbf{a}$ exists in $\mathbf{A}$ and belongs to $\mathbf{a}$ again,

(2)' there is a small subcategory $\mathbf{g}$ of $\mathbf{a}$ such that every object of $\mathbf{a}$ is a retract of a product of a family of objects from $\mathbf{g}$,

then every object of $\mathbf{A}$ has an $\mathbf{a}$-coresolution.

One obtains (1)' and (2)' by replacing the categories $\mathbf{A}$ and a, respectively, in (1) and (2) by the opposite categories $\mathbf{A}^{\mathrm{op}}$ and $\mathbf{a}^{\mathrm{op}}$. Given a functor $F: \mathbf{A} \longrightarrow \mathcal{A}$, where $\mathcal{A}$ is an abelian category and $F$ is linear (i.e., enriched in the category of abelian groups), its derived functors are defined to be the homology (respectively, cohomology) groups

$$
\begin{aligned}
\left(L_{n} F\right)(X) & :=\mathrm{H}_{n} F\left(A_{\bullet}\right), \\
\left(R^{n} F\right)(Y) & :=\mathrm{H}^{n} F\left(A^{\bullet}\right) .
\end{aligned}
$$

Here $A_{\bullet}$ is a resolution of $X$ and $A^{\bullet}$ a coresolution of $Y$.

We need the following concept of a $\Sigma$-algebra which allows the definition of a bigraded Ext-group.

6.4. Definition. A $\Sigma$-algebra $\mathbf{A}=(\mathbf{A}, \mathbf{a}, \Sigma)$ is an additive category $\mathbf{A}$ together with an additive subcategory $\mathbf{a}$ and an additive endofunctor $\Sigma: \mathbf{A} \rightarrow \mathbf{A}$ of $\mathbf{A}$ which carries $\mathbf{a}$ to $\mathbf{a}$ and which carries an a-resolution $A_{\bullet}$ of $X$ in $\mathbf{A}$ to an a-resolution $\Sigma A_{\bullet}$ of $\Sigma X$ in $\mathbf{A}$.

Dually, we define an $\Omega$-algebra $\mathbf{A}=(\mathbf{A}, \mathbf{a}, \Omega)$ where $\Omega$ carries an a-coresolution of $X$ in $\mathbf{A}$ to an $\mathbf{a}$-coresolution of $\Omega X$ in $\mathbf{A}$.

Given a $\Sigma$-algebra $\mathbf{A}$ and objects $X, Y$ in $\mathbf{A}$, we define the bigraded Ext-group by the cohomology

$$
\begin{aligned}
E_{2}^{s, t} & =\operatorname{Ext}_{\mathbf{A}}^{s}\left(\Sigma^{t} X, Y\right) \\
& =\operatorname{H}^{s} \operatorname{Hom}_{\mathbf{A}}\left(\Sigma^{t} A_{\bullet}, Y\right), \\
& =\operatorname{Ker}_{\operatorname{Hom}}\left(\Sigma^{t} \delta_{s+1}, Y\right) / \operatorname{Im}_{\operatorname{Hom}}\left(\Sigma^{t} \delta_{s}, Y\right) .
\end{aligned}
$$

Here $\Sigma^{t}=\Sigma \circ \ldots \circ \Sigma$ is the $t$-fold composite of $\Sigma$. Such groups appear in the $E_{2}$-term of the Adams spectral sequence.

\section{MAPPing ALGEBRAS}

In this section we consider topological analogues of $\Sigma$-algebras and $\Omega$-algebras of Definition 6.4, in order to provide a setting for defining higher order resolutions, and thus higher order derived functors.

7.1. Definition. Let $\mathbf{C}$ be a category enriched in pointed spaces. We call $\mathbf{C}$ a $\Sigma$ mapping algebra if the category $\mathbf{A}=\pi_{0} \mathbf{C}$ is a $\Sigma$-algebra and for each $n \geq 1$, the bifunctor $D_{n}: \mathbf{A}^{\mathrm{op}} \times \mathbf{A} \rightarrow \mathbf{A b}$, defined by $D_{n}(X, Y):=\pi_{n} \operatorname{Mor}_{\mathbf{C}}(X, Y)$, is equipped with a natural isomorphism:

$$
\tau_{\Sigma}: D_{n}(X, Y) \stackrel{\cong}{\rightarrow} \operatorname{Hom}_{\mathbf{A}}\left(\Sigma^{n} X, Y\right)
$$


for $X$ in a and $Y$ in $\mathbf{A}$. Here $\Sigma^{n}=\Sigma \circ \ldots \circ \Sigma$ is the $n$-fold composite of the endofunctor $\Sigma$ of $\mathbf{a}$.

Dually, $\mathbf{C}$ is an $\Omega$-mapping algebra if the category $\mathbf{A}=\pi_{0} \mathbf{C}$ is an $\Omega$-algebra and for each $n \geq 1, \quad D_{n}(X, Y):=\pi_{n} \operatorname{Mor}_{\mathbf{C}}(X, Y)$ is equipped with a natural isomorphism:

$$
\tau_{\Omega}: D_{n}(X, Y) \stackrel{\cong}{\rightarrow} \operatorname{Hom}_{\mathbf{A}}\left(X, \Omega^{n} Y\right)
$$

for $X$ in $\mathbf{A}$ and $Y$ in $\mathbf{a}$.

In the context of a pointed model category, such as $\mathbf{T o p}_{*}$, one should think of $\tau_{\Sigma}$ and $\tau_{\Omega}$ as being related by the adjunction of $\Omega$ and $\Sigma$.

7.2. Definition. A $\Sigma$-mapping algebra $\mathbf{C}$ is complete if the endofunctor $\Sigma$ of $\mathbf{A}=\pi_{0} \mathbf{C}$ is induced by an endofunctor $\Sigma$ of $\mathbf{C}$ and we have a binatural transformation

$$
\tau_{\Sigma}: \operatorname{Mor}_{\mathbf{C}}(\Sigma A, Y) \rightarrow \Omega \operatorname{Mor}_{\mathbf{C}}(A, Y),
$$

where we use the topological loop space functor on pointed spaces. We require the functor $\Sigma: \mathbf{C} \longrightarrow \mathbf{C}$ to preserve zero maps and coproducts in $\mathbf{C}$.

Dually, an $\Omega$-mapping algebra $\mathbf{C}$ is complete if the endofunctor $\Omega$ of $\mathbf{A}=\pi_{0} \mathbf{C}$ is induced by an endofunctor $\Omega$ of $\mathbf{C}$ and we have a binatural transformation

$$
\tau_{\Omega}: \operatorname{Mor}_{\mathbf{C}}(Y, \Omega A) \rightarrow \Omega \operatorname{Mor}_{\mathbf{C}}(Y, A) .
$$

Again we require that the functor $\Omega: \mathbf{C} \rightarrow \mathbf{C}$ preserve zero maps and products in C. Iteration of $\tau_{\Sigma}$ (respectively, $\tau_{\Omega}$ ) induces the isomorphisms $\tau_{\Sigma}$ (respectively, $\left.\tau_{\Omega}\right)$ in Definition 7.1 .

7.3. Example. There are a number of different simplicial model categories of spectra, including the simplicial spectra of [BoF], the $S$-modules of [EKMM], the symmetric spectra of [HSS], and the orthogonal spectra of [MMSS]. All of these are naturally pointed; in this and later sections, we let Spec be any category of spectra which is enriched in pointed topological spaces (or simplicial sets), with pointed function spaces

$$
\operatorname{Mor}_{\text {Spec }}(X, Y)=\operatorname{Map}(X, Y) \quad \text { for } X \text { and } Y \text { in Spec. }
$$

We always assume that $X$ and $Y$ are both fibrant and cofibrant in our chosen model category.

Clearly zero maps $o: X \rightarrow Y$ are defined in Spec. Let $\mathcal{X}$ be a class of objects in Spec closed under coproducts and the suspension $\Sigma$ : that is, for $A, A^{\prime} \in \mathcal{X}$ we have $A \vee A^{\prime}, \Sigma A \in \mathcal{X}$. Then we have $\{\mathcal{X}\} \subset$ Spec, where $\{\mathcal{X}\}$ is the full subcategory in Spec with objects in $\mathcal{X}$. In this case $\mathbf{C}=\mathbf{S p e c}$, with $\mathbf{a}=\pi_{0}\{\mathcal{X}\} \subset \mathbf{A}=\pi_{0} \mathbf{C}$, is a complete $\Sigma$-mapping algebra.

Dually, let $\mathcal{Y}$ be a class of objects in spec such that $\mathcal{Y}$ is closed under products and loop functor $\Omega$, that is, for $B, B^{\prime} \in \mathcal{Y}$, we have $B \times B^{\prime}, \Omega B \in \mathcal{Y}$. Then we have

$$
\{\mathcal{Y}\} \subset \text { Spec }
$$

where $\{\mathcal{Y}\}$ is the full subcategory in Spec with objects in $\mathcal{Y}$. Then $\mathbf{C}=$ Spec with $\mathbf{a}=\pi_{0}\{\mathcal{Y}\} \subset \mathbf{A}=\pi_{0} \mathbf{C} \quad$ is a complete $\Omega$-mapping algebra.

7.4. Example. Let $p$ be a prime and let $H=\mathrm{HF}_{p}$ be the $\bmod p$ Eilenberg-Mac Lane spectrum. Let $\mathcal{Y}$ be given by all products

$$
\Omega^{n_{1}} H \times \Omega^{n_{2}} H \times \ldots \times \Omega^{n_{k}} H
$$


with $n_{i} \geq 0 \quad$ for $i=1, \ldots, k \quad(k \geq 0)$. Then $\mathbf{C}=$ Spec with $\mathbf{a}=\pi_{0}\{\mathcal{Y}\} \quad$ is a complete $\Omega$-mapping algebra, which we call the Eilenberg-Mac Lane mapping algebra. This is used in the Adams spectral sequence.

7.5. Remark. In the examples of mapping algebras above the category $\mathbf{C}=\mathbf{S p e c}$ is very large. For computations, however, we consider only the mapping algebras $\mathbf{C}^{\prime}$ which are generated by $\{\mathcal{X}\} \quad$ (respectively, $\{\mathcal{Y}\}$ ) and two further objects $X$ and $Y$ in Spec.

\section{Higher order RESOLUtions}

We can use the definitions of Section 7 to define higher order resolutions, and state our main results about them, which will be proved in Section 14.

These higher order resolutions will be used in Section 15 to describe the higher terms of the Adams spectral sequence, providing an alternative way of setting up this spectral sequence, avoiding the classical topological construction of [A] using a tower of (co)fibrations of spectra.

8.1. Definition. Let $\mathbf{C}$ be a $\Sigma$-mapping algebra with $\mathbf{a} \subset \mathbf{A}=\pi_{0} \mathbf{C}$. An $n$-th order chain complex

$$
K: \mathbb{Z}(\infty,-1)_{\otimes}^{n} \longrightarrow \mathrm{Nul}_{n} \mathbf{C}
$$

is called $n$-th order resolution of $X$ in $\mathrm{Nul}_{n} \mathbf{C}$ if it is based on an a-resolution in $\mathbf{A}$,

$$
A_{\bullet}=\left(\ldots \stackrel{\delta_{2}}{\longrightarrow} A_{1} \stackrel{\delta_{1}}{\longrightarrow} A_{0} \stackrel{\delta_{0}}{\longrightarrow} A_{-1}\right) \text {, }
$$

of $X=A_{-1}$.

8.2. Resolution Theorem. Given an $\mathbf{a}$-resolution $A_{\bullet}$ of $X$ in $\mathbf{A}$, there is an $n$-th order resolution $K$ of $X$ based on $A_{\bullet} \quad$ in $\operatorname{Nul}_{n} \mathbf{C} \quad(n \geq 1)$.

This will follow from the more general Theorem 14.5 below.

8.3. Remark. The Theorem shows that, if 'minimal' a-resolutions exist (as in the case of the Adams spectral sequence), then also an $n$-th order minimal resolution exists which is based on a minimal resolution in $\mathbf{A}$. This is of high importance for computations.

8.4. Definition. Dually, let $\mathbf{C}$ be a $\Omega$-mapping algebra with $\mathbf{a} \subset \mathbf{A}=\pi_{0} \mathbf{C}$. If an $n$-th order chain complex

$$
L: \mathbb{Z}(+1,-\infty)_{\otimes}^{n} \longrightarrow \mathrm{Nul}_{n} \mathbf{C}
$$

is based on an a-coresolution in $\mathbf{A}$

$$
A^{\bullet}=\left(A_{1} \stackrel{\delta_{1}}{\longrightarrow} A_{0} \stackrel{\delta_{0}}{\longrightarrow} A_{-1} \stackrel{\delta_{-1}}{\longrightarrow} \ldots\right)
$$

with $A_{1}=Y$, we say that $L$ is an $n$-th order coresolution of $X$ in $\operatorname{Nul}_{n} \mathbf{C}$.

8.5. Dual Resolution Theorem. Given an $\mathbf{a}$-coresolution $A^{\bullet}$ of $Y$ in $\mathbf{A}$, there is an $n$-th order coresolution $L$ of $Y$ based on $A^{\bullet} \quad$ in $\operatorname{Nul}_{n} \mathbf{C} \quad(n \geq 1)$.

This will follow from Theorem 14.6 below.

8.6. Remark. In view of Lemma 3.6 (a) in [BJ2], a first-order resolution in $\mathrm{Nul}_{1} \mathbf{C}$ is a secondary resolution in the sense of [BJ2]. 


\section{LEFT CUBicAL BALLS}

The higher order resolutions of Theorems 8.2 and 8.5 are built of left cubes in a mapping algebra $\mathbf{C}$. The main goal of this paper is to elucidate those properties of left cubes which are actually needed for the construction of these resolutions, which are used in turn to calculate the differentials in the Adams spectral sequence.

The relevant properties will be described using the new notion of a left cubical ball, which we introduce in this section. Such balls serve as a book-keeping device to describe the combinatorics of higher tracks, and allow us to define certain associated obstructions in homotopy groups of mapping spaces in $\mathbf{C}$ in the next section.

9.1. Definition. A ball of dimension $n$ is a finite regular CW-complex $B$ with a subcomplex $\partial B$ and a homeomorphism of pairs

$$
\left(E^{n}, S^{n-1}\right) \approx(B, \partial B)
$$

where $E^{n}$ is the Euclidean ball. We do not assume that $B$ has only one $n$-cell. Two balls $B$ and $B^{\prime}$ are equivalent if there is a cellular isomorphism $B \approx B^{\prime}$. A ball $B$ is a union

$$
B=B_{1} \cup \ldots \cup B_{k}
$$

of closed $n$-cells $\quad B_{i} \quad$ in $B$. We say that $A$ is a sub-ball of $B$ if $A=B_{i_{1}} \cup \ldots \cup B_{i_{t}}$ for $1 \leq i_{1}<\ldots<i_{t} \leq k$ is a ball and if for $t<k$, the closure of the complement $B \backslash A$ in $B$ is also a ball, denoted by $A_{B}$, so that $B=A \cup A_{B}$. We also require that $S:=A \cap A_{B}$ be a ball of dimension $n-1$, contained in both $\partial A$ and $\partial A_{B}$, with the closures of $\partial A \backslash S$ in $\partial A$ and of $\partial A_{B} \backslash S$ in $\partial A_{B}$ likewise balls of dimension $n-1$.

If $A$ is also a sub-ball of a ball $C$ with $S=A \cap A_{B}=A \cap A_{C}$, then we obtain the union of complements

$$
A_{B} \cup A_{C}=A_{B} \cup_{s} A_{C},
$$

which is also a ball of dimension $n$, being the union of two $n$-balls along $(n-1)$-balls $S$ in their boundaries (identified with opposite orientations on $S$ ). See Figure 2 .

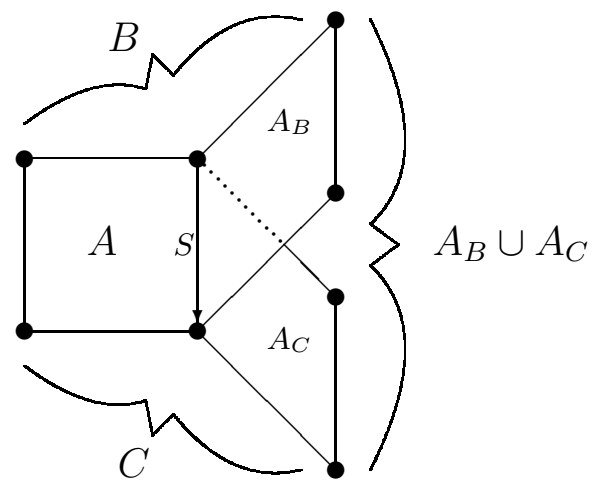

FiguRE 2. Union of complements

9.2. Example. Let $T_{0}^{n}$ be the union of all cells $I^{i-1} \times\{0\} \times I^{n+1-i}$ in $I^{n+1}$, and let $T_{1}^{n}$ be the union of all cells $I^{i-1} \times\{1\} \times I^{n+i-1} \quad(i=1, \ldots, n+1)$. Thus $T_{\varepsilon}^{n}$ is the corner of $I^{n+1}$ consisting of all $n$-facets touching the vertex $(\varepsilon, \varepsilon, \ldots, \varepsilon)$ $(\varepsilon=0,1)$, and $T_{0}^{n}$ and $T_{1}^{n}$ are balls of dimension $n$, with $n+1$ closed $n$-cells. 
9.3. Definition. A left cubical ball is a ball $B$ with a 0 -vertex $0 \in B-\partial B$ equipped with the following:

(1) Each closed $n$-cell $B_{i}$ has a homeomorphism $h_{i}: I^{n} \rightarrow B_{i}$.

(2) Each closed $(n-1)$-cell $\bar{e}$ has a homeomorphism $h_{e}: I^{n-1} \rightarrow \bar{e}$. such that if $\bar{e} \subset B_{i} \cap B_{j}$, the diagram

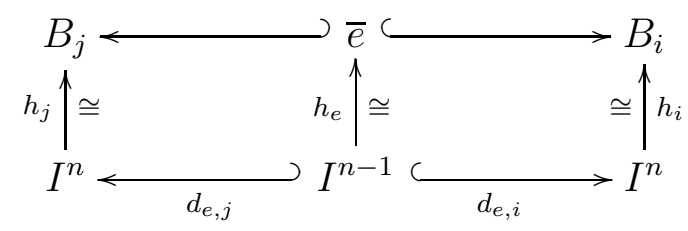

commutes.

Here $d_{e, j}$ and $d_{e, i}$ are morphisms in the left cubical category $\bar{\square}$. The vertex 0 is also a vertex of each $B_{i}$ and the equivalence $h_{i}: I^{n} \approx B^{i}$ carries 0 to 0 . Moreover, the union

$$
h_{1}\left(T_{1}^{n-1}\right) \cup \ldots \cup h_{k}\left(T_{1}^{n-1}\right)=\partial B
$$

is the boundary of $B$.

9.4. Example. The $n$-ball $T_{0}^{n}$ of Example 9.2 is a left cubical ball, as is the pushout of $I^{n} \longleftarrow T_{0}^{n-1} \longrightarrow I^{n}$, called the double of $I^{n}$.

Further examples of left cubical balls of dimension 2 appear in Figure 1 above and in Figure 3 ,
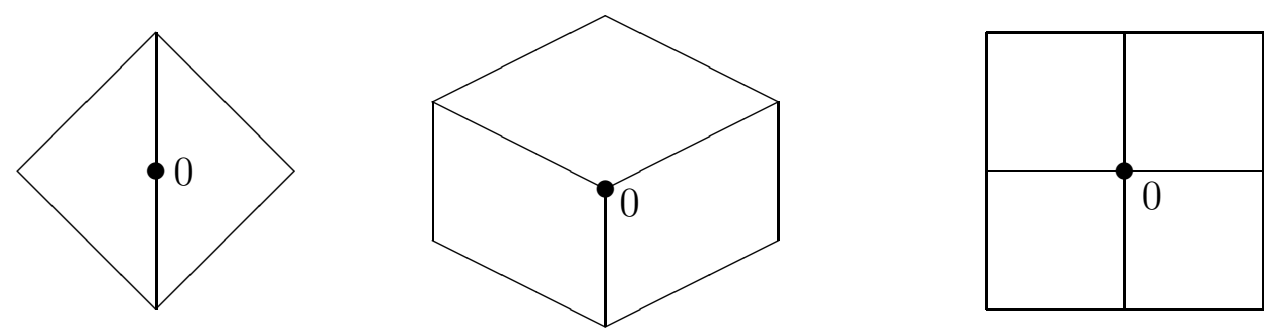

Figure 3. Some left cubical balls of dimension 2

9.5. Lemma. Let $A$ be a sub-ball of $B$ and $C$, where $B$ and $C$ are left cubical; then the union of complements $A_{B} \cup A_{C}$ is left cubical.

9.6. Remark. Let $B$ be a left cubical ball of dimension $n$ with $k$ closed $n$-cells. Then $B$ is equivalent to the double of $I^{n}$ for $k=2$ and $B$ is equivalent to $T_{0}^{n}$ for $k=n+1$. For $2<k<n+1$, such a ball does not exist. For $k \geq n+1$, there is a one-to-one correspondence between left cubical balls (up to equivalence) and simplicial complexes homeomorphic to the $(n-1)$-sphere $S^{n-1}$. The correspondence carries $B$ to the boundary of a small neighborhood of 0 in $B$, where the link of the corner of an $n$-cube forms an $n-1$-simplex. Thus in Figure 1, the eight 2-cubes yield an octagon (a triangulation of the 1-sphere). 


\section{Obstructions}

We now describe, for a general space $X$, the type of obstructions derived from cubical balls, which we shall later need in the case when $X$ is a mapping space.

10.1. Definition. Let $X$ be a pointed space with base point $o \in X$, let $B$ be a ball, and let $a: B \longrightarrow X$ be a map with $a(\partial B)=o$. We obtain the map

$$
a: S^{n} \approx E^{n} / S^{n-1} \approx B / \partial B \longrightarrow X,
$$

which represents an element $\mathcal{O}(a) \in \pi_{n}(X)$ in the $n$-th homotopy group of $X$. Now let $B=B_{1} \cup \ldots \cup B_{k}$ be a left cubical ball. Then

$$
I^{n} \stackrel{h_{i}}{\rightarrow} B_{i} \subset B \stackrel{a}{\rightarrow} X
$$

is a left $n$-cube representing an $n$-track $a_{i} \in \operatorname{Nul}_{n}(X)_{n}$.

Then for $\bar{e} \subset B_{i} \cap B_{j}$ we have the gluing condition in $B$ (see Definition 9.3).

$$
d_{e, i}^{*} a_{i}=d_{e, j}^{*} a_{j} .
$$

A $k$-tuple $\left(a_{1}, \ldots, a_{k}\right)$ of $n$-tracks $a_{i}$ in $\operatorname{Nul}_{n}(X)_{n}$ satisfying (10.3) is called a left $n$-cubical ball in $X$.

10.4. Lemma. A left $n$-cubical ball $\left(a_{1}, \ldots, a_{k}\right)$ in $X$ yields a map $a: B \rightarrow X$ with $a(\partial B)=o$, well-defined up to homotopy relative to the boundary. This defines the obstruction $\mathcal{O}_{B}\left(a_{1}, \ldots, a_{k}\right)=\mathcal{O}(a)$ in $\pi_{n}(X)$ as above.

Now let $B=T_{0}^{n}=B_{1} \cup \ldots \cup B_{n+1} \quad$ be the ball of $\$ 9.2$, and let $a_{1}, \ldots, a_{n+1} \in$ $\operatorname{Nul}_{n}(X)_{n}$ be $n$-tracks satisfying the gluing condition (10.3). Then we get the boundary property:

10.5. Lemma. $\mathcal{O}_{T_{0}^{n}}\left(a_{1}, \ldots, a_{n+1}\right)=0 \quad$ if and only if there exist $\bar{a} \in \operatorname{Nul}_{n+1}(X)_{n+1}$ with $\partial^{i} \bar{a}$ representing $a_{i}$.

Proof. We choose representatives $a_{i}^{\prime}$ of $a_{i}$ which define a map

$$
\overline{\bar{a}}: \partial I^{n+1} \longrightarrow X
$$

with $\overline{\bar{a}}\left(T_{1}^{n}\right)=0$ and $\left.\overline{\bar{a}}\right|_{T_{0}^{n}}=a_{1}^{\prime} \cup \ldots \cup a_{n+1}^{\prime}$. Here $\overline{\bar{a}}$ extends to $I^{n+1}$ if and only if $\mathcal{O}\left(a_{1}^{\prime} \cup \ldots \cup a_{n+1}^{\prime}\right)=0$.

The next result is the Complement Rule.

10.6. Lemma. Let $B=A_{1} \cup \ldots \cup A_{r} \cup B_{1} \cup \ldots \cup B_{t}$ and $C=A_{1} \cup \ldots \cup A_{r} \cup C_{1} \cup \ldots \cup C_{s}$ be left cubical balls with a common sub-ball $A=A_{1} \cup \ldots \cup A_{r}$. Then

$$
\mathcal{O}_{C}\left(a_{1}, \ldots, a_{r}, c_{1}, \ldots c_{s}\right)=0
$$

implies that for $D=A_{B} \cup A_{C}$ as in $\$ 9.1$ we have:

$$
\mathcal{O}_{B}\left(a_{1}, \ldots, a_{r}, b_{1}, \ldots, b_{t}\right)=\mathcal{O}_{D}\left(b_{1}, \ldots, b_{t}, c_{1}, \ldots, c_{s}\right) .
$$

Proof. This follows from the Homotopy Addition Theorem (cf. [M]) and our conventions on the union of complements (Definition 9.1).

Of course, there is the following Double Rule: 
10.7. Lemma. If $B=B_{1} \cup B_{2}$ is the double of $I^{n}$ then for $a_{1}=a_{2}$ we have:

$$
\mathcal{O}_{B}\left(a_{1}, a_{2}\right)=0 \text {. }
$$

10.8. Definition. Let $B=B_{1} \cup \ldots \cup B_{k}$ be a left cubical ball. Then for each $1 \leq i \leq k \quad$ we have a map

$$
\bar{\varepsilon}_{i}: I^{n} \approx B_{i} \subset B \approx E^{n},
$$

where $I^{n}$ and $E^{n}$ are oriented by the inclusions of $I^{n}$ and $E^{n}$ in $\mathbb{R}^{n}$. We set $\varepsilon_{i}=+1$ if the map $\bar{\varepsilon}_{i}$ is orientation preserving, otherwise $\varepsilon_{i}=-1$. We call $\varepsilon_{i}$ the orientation sign of $B_{i}$.

Let $B=B_{1} \cup B_{2}$ be the double of $I^{n}$. Then $\varepsilon_{1}=-\varepsilon_{2}$, and we can choose $B_{1}$ so that $\varepsilon_{1}=1$, In this case we define the action + of $\alpha \in \pi_{n}(X)$ on an $n$-track $a \in \operatorname{Nul}_{n}(X)_{n} \quad(n \geq 1) \quad$ by letting $a+\alpha$ denote the unique $n$-track $b$ such that $\mathcal{O}(b, a)=\alpha$. This can of course be described explicitly in terms of the coaction of the $n$-sphere on the $n$-ball.

10.9. Lemma. The action + yields a well defined effective and transitive action of the group $\pi_{n}(X)$ on the set of all n-tracks $a \in \operatorname{Nul}_{n}(X)_{n}$ which coincide on the boundary (that is, $\partial^{i} a=b_{i}$, where $\left(b_{1}, \ldots, b_{n}\right)$ is fixed).

10.10. Lemma. Let $B=B_{1} \cup \ldots \cup B_{k}$ be a left cubical ball and let $\mathcal{O}_{B}\left(a_{1}, \ldots, a_{k}\right)$, $\mathcal{O}_{B}\left(a_{1}^{\prime}, \ldots, a_{k}^{\prime}\right)$ be defined, where

$$
\begin{cases}a_{i}^{\prime}=a_{i} & \text { for } i \neq j \\ a_{j}^{\prime}=a_{j}+\alpha & \text { for } i=j, \alpha \in \pi_{n}(X) .\end{cases}
$$

Then we have the Action Formula:

$$
\mathcal{O}\left(a_{1}^{\prime}, \ldots, a_{k}^{\prime}\right)=\mathcal{O}\left(a_{1}, \ldots, a_{k}\right)+\varepsilon_{j} \alpha .
$$

\section{The Algebra of LEFT $n$-CUBiCAL BALLS}

In this section we collect together those properties of left $n$-cubical balls in the mapping spaces of a mapping algebra $\mathbf{C}$ which are needed for the construction of higher order resolutions. These properties are encoded in the notion of the algebra $(\mathbf{T}, \mathbf{A}, D, \mathcal{O})$ of left $n$-cubical balls in $\mathbf{C}$. The main example we have in mind is given as follows:

Let $\mathbf{C}$ be a category enriched in pointed spaces. Let $n \geq 1$ and let $\mathbf{T}=\mathrm{Nul}_{n} \mathbf{C}$ and $\mathbf{A}=\pi_{0} \mathbf{C}$. We define a functor $D: \mathbf{A}^{\mathrm{op}} \times \mathbf{A} \longrightarrow \mathbf{A b}$ by $D(X, Y):=$ $\pi_{n} \operatorname{Mor}_{\mathbf{C}}(X, Y)$, so $\mathcal{O}_{B}\left(a_{1}, \ldots, a_{k}\right)$ is defined in $\operatorname{Nul}_{n} \operatorname{Mor}_{\mathbf{C}}(X, Y)$ as in (10.2).

For $n=1$ we assume that the group $\pi_{1} \operatorname{Mor}_{\mathbf{C}}(X, Y)$ is abelian for all objects $X$, $Y$ in $\mathbf{C}$.

As shown in Section 10, the system $(\mathbf{T}, \mathbf{A}, D, \mathcal{O})$ is a special case of the following abstract concept:

11.1. Definition. An algebra of left $n$-cubical balls $(n \geq 1)$ is a system $\mathbf{T}=$ $(\mathbf{T}, \mathbf{A}, D, \mathcal{O})$ given by an $n$-graded category $\mathbf{T}$, a quotient functor $\mathbf{T}^{0} \longrightarrow \mathbf{A} ; \quad$ a bifunctor $D: \mathbf{A}^{\mathrm{op}} \times \mathbf{A} \longrightarrow \mathbf{A b}$, and an obstruction operator $\mathcal{O}$. The following properties are required to hold: 
(1) $\mathbf{T}$ is enriched in left $n$-cubical sets and has zero maps, that is, for all objects $X, Y$ in $\mathbf{T}$, we have the $n$-cubical set $\operatorname{Mor}_{\mathbf{T}}(X, Y)$ with operators $\left(d_{o}^{i}\right)^{*}=\partial^{i}$ and zero elements $o^{t} \in \operatorname{Mor}_{\mathbf{T}}(X, Y)_{t}$ such that

$$
\begin{array}{r}
\partial^{i}(f g)=\left(\partial^{i} f\right) g \text { for } i \leq \operatorname{dim}(f) \\
\partial^{i}(f g)=f\left(\partial^{i-\operatorname{dim}(f)} g\right) \text { for } i>\operatorname{dim}(f) \\
o^{t} g=o^{t+\operatorname{dim}(g)} \\
f o^{h}=o^{\operatorname{dim}(f)+h} .
\end{array}
$$

Here $f g$ is the composite in the $n$-graded category $\mathbf{T}$, which is defined if $\operatorname{dim}(f)+\operatorname{dim}(g) \leq n$.

(2) The 0-skeleton $\mathbf{T}^{0}$ is the subcategory of $\mathbf{T}$ consisting of morphisms $f$ with $\operatorname{dim}(f)=0$, this is a category together with a functor $q: \mathbf{T}^{0} \longrightarrow \mathbf{A}$ which is the identity on objects and full (a quotient functor). Moreover, $D$ is a bifunctor

$$
D: \mathbf{A}^{\mathrm{op}} \times \mathbf{A} \longrightarrow \mathbf{A b}
$$

into the category of abelian groups. Here $D$ defines via $q$ a bifunctor on $\mathbf{T}^{0}$ which satisfies $\left(o^{0}\right)^{*}=o$ and $\left(o^{0}\right)_{*}=o$. For a zero map $o^{0}: X \longrightarrow Y$ in $\mathbf{T}^{0} \quad$ we obtain the zero map $o_{X, Y}=q\left(o^{0}\right)$ in $\mathbf{A}$.

For $f: X \longrightarrow Y$ in $\mathbf{T}^{0}$, we have $q(f)=o_{X, Y} \quad$ if and only if there is $F: X \longrightarrow Y$ in $\mathbf{T}$ with $\operatorname{dim}(F)=1$ and $\partial^{1} F=f$. This is the boundary property in dimension 1.

(3) The obstruction operator $\mathcal{O}$ yields for each left cubical ball $B$ an element

$$
\mathcal{O}_{B}\left(a_{1}, \ldots, a_{k}\right) \in D(X, Y)
$$

where $a_{1}, \ldots, a_{k} \in \operatorname{Mor}_{\mathbf{T}}(X, Y)_{n}$ is a $k$-tuple satisfying the gluing condition in $B$, see (10.3).

This obstruction operator satisfies the complement rule, the double rule, and the action formula as in Section 10. Here the action + of $D(X, Y)$ on the set $\operatorname{Mor}_{\mathbf{T}}(X, Y)_{n}$ is defined by:

$$
\text { if } \mathcal{O}_{B}(b, a)=\alpha \text {, then } b=a+\alpha .
$$

This $b$ is required to be unique. Here $B$ is the double of $I^{n}$ with $\varepsilon_{1}=+1$.

The action + is transitive and effective on the set of all elements $a$ in $\operatorname{Mor}_{\mathbf{T}}(X, Y)_{n}$ which coincide on the boundary (that is, $\partial^{i} a=b_{i}$, where $\left(b_{1}, \ldots, b_{n}\right)$ is fixed).

(4) The obstruction operator satisfies for

$$
f \in \operatorname{Mor}_{\mathbf{T}}\left(X^{\prime}, X\right)_{0} \text { and } g \in \operatorname{Mor}_{\mathbf{T}}\left(Y, Y^{\prime}\right)_{0}
$$

the naturality rule

$$
\begin{aligned}
\mathcal{O}_{B}\left(g a_{1}, \ldots, g a_{k}\right) & =g_{*} \mathcal{O}_{B}\left(a_{1}, \ldots, a_{k}\right) \\
\mathcal{O}_{B}\left(a_{1} f, \ldots, a_{k} f\right) & =f^{*} \mathcal{O}_{B}\left(a_{1}, \ldots, a_{k}\right) .
\end{aligned}
$$

Here $f^{*}$ and $g_{*}$ denote the induced maps on $D$. This implies that $g(a+\alpha)=g a+g_{*} \alpha$ and $(a+\alpha) f=a f+f^{*} \alpha$. 
(5) The obstruction operator satisfies the following triviality rule: For morphisms

$$
Z \stackrel{f}{\longleftarrow} Y \stackrel{g}{\longleftarrow} X
$$

in $\mathbf{T}$ with $\operatorname{dim}(f), \operatorname{dim}(g) \leq n$ and

$$
\operatorname{dim}(f)+\operatorname{dim}(g)=n+1
$$

we have the $(n+1)$-tuple $\left(a_{1}, \ldots, a_{n+1}\right)$ in $\operatorname{Mor}_{\mathbf{T}}(X, Z)_{n}$ given by

$$
a_{t}= \begin{cases}\left(\partial^{t} f\right) g & \text { for } 1 \leq t \leq \operatorname{dim}(f) \\ f\left(\partial^{t-\operatorname{dim}(f)} g\right) & \text { for } \operatorname{dim}(f)<t \leq n+1\end{cases}
$$

This $(n+1)$-tuple satisfies the gluing condition in $B=\mathbf{T}_{0}^{n}$. The associated obstruction

$$
\mathcal{O}_{B}\left(a_{1}, \ldots, a_{n+1}\right)=0
$$

is trivial.

11.3. Remark. The case $n=1$ is classical, and is considered in the next section.

We now generalize the concept of an $n$-th order chain complex (defined in Section 4 for a mapping algebra $\mathbf{C}$ ), replacing $\mathrm{Nul}_{n} \mathbf{C}$ by an abstract algebra of left $n$-cubical balls $\mathbf{T}$ :

11.4. Definition. Let $(\mathbf{T}, \mathbf{A}, D, \mathcal{O})$ be an algebra of left $n$-cubical balls. A functor of $n$-graded categories

$$
K: \mathbb{Z}(N, M)_{\otimes}^{n} \rightarrow \mathbf{T}
$$

satisfying the inclusion property (4.2) is an $n$-th order pre-chain complex in $\mathbf{T}$. This is an $n$-th order chain complex in $\mathbf{T}$ if for $i, i-n-2 \in \mathbb{Z}(N, M)$, the obstructions

$$
\mathcal{O} K\left(i, \partial I_{n+1}\right)=\mathcal{O}_{B}\left(b_{1}, \ldots, b_{n+1}\right)=0
$$

vanish. Here $B$ is the left cubical ball $B=\mathbf{T}_{0}^{n}$, and

$$
K\left(i, \partial I_{n+1}\right)=\left\{\begin{array}{l}
b_{1}=K\left(i, \emptyset \otimes I_{n}\right) \\
b_{r+1}=K\left(i, I_{r} \otimes I_{n-r}\right) \\
b_{n+1}=K\left(i, I_{n} \otimes \emptyset\right)
\end{array} \quad \text { for } 1 \leq r \leq n-1\right.
$$

(see (4.7)). Since $K$ is a functor we have

$$
\begin{aligned}
& K\left(i, \emptyset \otimes I_{n}\right)=K(i-n-1, \emptyset) K\left(i, I_{n}\right)=\delta_{i-n-1} K\left(i, I_{n}\right) \\
& K\left(i, I_{r} \otimes I_{s}\right)=K\left(i-s-1, I_{r}\right) K\left(i, I_{s}\right) \\
& K\left(i, I_{n} \otimes \emptyset\right)=K\left(i-1, I_{n}\right) K(i, \emptyset)=K\left(i-1, I_{n}\right) \delta_{i}
\end{aligned}
$$

where the right hand side denotes composition in $\mathbf{T}$. We define higher order Toda brackets in $\mathbf{T}$

$$
\left\langle\delta_{1}, \ldots, \delta_{n+2}\right\rangle \subset D\left(K_{n+2}, K_{0}\right)
$$

as in Definition 4.8 . 


\section{TRACK CATEgORIES AND ALGEBRAS OF LEFT 1-CUBICAL BALLS}

In this section we discuss algebras of left $n$-cubical balls in the special case $n=1$. We show that every abelian track category (cf. [BW]) has the structure of an algebra of left 1 -cubical balls. This shows that algebras of left $n$-cubical balls are $n$-dimensional analogues of track categories for every $n \geq 1$.

12.1. Definition. A track category is a category $\mathbf{C}$ enriched in groupoids. For objects $X, Y$ in $\mathbf{C}$ we have the groupoid $\operatorname{Mor}_{\mathbf{C}}(X, Y)$ with objects $f, g$ and morphisms $F: f \longrightarrow g$.

The morphisms $F: f \longrightarrow f$ form the automorphism group $\operatorname{Aut}_{\mathbf{C}}(f)$, and we write $f \simeq g$ if there is $F: f \longrightarrow g$. Let $\operatorname{dim}(f)=0, \quad \operatorname{dim}(F)=1, \quad\left(d_{0}^{1}\right)^{*} F=f$, and $\left(d_{1}^{1}\right)^{*} F=g$. Morphisms of dimension 0 form the category $\mathbf{C}_{0}$, and the homotopy relation $\simeq$ defines the homotopy category

$$
\mathbf{A}=\pi_{0} \mathbf{C}=\mathbf{C}_{0} / \simeq
$$

The track category $\mathbf{C}$ is called abelian if all automorphism groups $\operatorname{Aut}_{\mathbf{C}}(f)$ are abelian groups. We assume that $\mathbf{C}$ has zero maps $o_{X, Y} \in \operatorname{Mor}_{\mathbf{C}}(X, Y)_{0}$ for each $X, Y \in \mathbf{C}$. We then obtain a bifunctor $D: \mathbf{A}^{\mathrm{op}} \times \mathbf{A} \rightarrow \mathbf{A b}$ defined

$$
D(X, Y):=\operatorname{Aut}_{\mathbf{C}}\left(o_{X, Y}\right) \text {. }
$$

We define the 1-graded category $\mathbf{T}$ associated to $\mathbf{C}$ (cf. \$2.1) by

$$
\operatorname{Mor}_{\mathbf{T}}(X, Y)_{i}:= \begin{cases}\operatorname{Mor}_{\mathbf{C}}(X, Y)_{0} & \text { if } i=0 \\ \left\{(F, f), F: f \rightarrow o_{X, Y}\right\} \subset \operatorname{Mor}_{\mathbf{C}}(X, Y)_{1} & \text { if } i=1 .\end{cases}
$$

Let $\partial^{1}$ be defined by $\partial^{1}(F, f)=f$, and let the zero elements be given by $o^{0}=o_{X, Y}$, $o^{1}=$ identity of $o_{X, Y}$.

12.2. Proposition. For any abelian track category $\mathbf{C}$ with zero maps, $\operatorname{Nul}_{1}(\mathbf{C})=$ $(\mathbf{T}, \mathbf{A}, D, \mathcal{O})$ with $\mathbf{T}, \mathbf{A}$ and $D$ as above and with the obstruction operator $\mathcal{O}$ as follows is an algebra of left 1-cubical balls.

Up to equivalence there is only one left cubical ball $B$ of dimension 1: this is the double of $I$, which is equivalent to $T_{0}^{1}$. Given $a_{1}=(F, f)$ and $a_{2}=(G, g)$ with gluing condition $\partial^{1} a_{1}=f=g=\partial^{1} a_{2}$, let

$$
\mathcal{O}_{B}\left(a_{1}, a_{2}\right):=F G^{-1} \in \operatorname{Aut}_{\mathbf{C}}\left(o_{X, Y}\right)
$$

be the obstruction. The action for $\alpha \in \operatorname{Aut}_{\mathbf{C}}\left(o_{X, Y}\right)$ and $a=(F, f)$ is given by $a+\alpha=(\alpha F, f)$, with $\mathcal{O}_{B}(a+\alpha, a)=\alpha F F^{-1}=\alpha$. The triviality rule of $\mathcal{O}$ is satisfied, since for a diagram

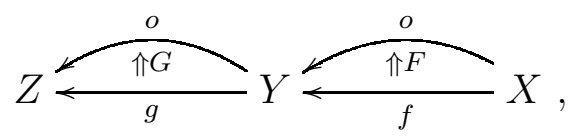

in $\mathbf{C}$ we have the formula $G f=g F$ by the usual interchange law (since $o_{Y, Z} F=$ $\left.G o_{X, Y}=\operatorname{Id}_{o_{X, Z}}\right)$, so that

$$
\mathcal{O}_{B}\left(a_{1}, a_{2}\right)=o \quad \text { for } \quad a_{1}=G\left(\partial^{1} F\right) \text { and } a_{2}=\left(\partial^{1} G\right) F .
$$


12.3. Example. Let $\mathbf{C}$ be a category enriched in groupoids with zero maps and let $\mathbf{C}$ be abelian. Then the algebra of left 1-cubical balls $\mathrm{Nul}_{1}(\mathbf{C})$ is defined and a triple Toda bracket

$$
\left\langle\delta_{1}, \delta_{2}, \delta_{3}\right\rangle \text { in } \mathrm{Nul}_{1}(\mathbf{C})
$$

coincides with the classical triple Toda bracket in C. Moreover, a 1-st order chain complex in $\mathrm{Nul}_{1}(\mathbf{C})$ as defined in $\$ 11.4$ coincides with a secondary chain complex in $\mathrm{C}$ as studied in [BJ2].

12.4. Remark. Abelian track categories are classified by cohomology, see $[\mathrm{BW}, \mathrm{BD}, \mathrm{P}$, B1, BJ1. It would be interesting to classify accordingly algebras of left $n$-cubical balls for $n \geq 1$.

\section{The inductive Step of the Resolution theorem}

Resolution Theorem 8.2 refers to higher order resolutions in a mapping algebra. In the following section we shall prove a more general resolution theorem, for certain types of algebras of left $n$-cubical balls, which we now define. The proof is by induction, and we give the induction step here.

13.1. Definition. An algebra of left $n$-cubical balls $\mathbf{T}=(\mathbf{T}, \mathbf{A}, D, \mathcal{O})$ is a $\Sigma$-algebra of left $n$-cubical balls if $\mathbf{A}=(\mathbf{A}, \mathbf{a}, \Sigma)$ is a $\Sigma$-algebra (cf. \$6.4 $)$ and

$$
D(X, Y)=\operatorname{Hom}_{\mathbf{A}}\left(\Sigma^{n} X, Y\right)
$$

for $X$ in $\mathbf{a}$ and $Y$ in $\mathbf{A}$. We say that $\mathbf{T}$ is an $\Omega$-algebra of left $n$-cubical balls if $\mathbf{A}=(\mathbf{A}, \mathbf{a}, \Omega)$ is an $\Omega$-algebra (cf. \$‥4) and

$$
D(X, Y)=\operatorname{Hom}_{\mathbf{A}}\left(X, \Omega^{n} Y\right)
$$

for $Y$ in a and $X$ in $\mathbf{A}$.

13.2. Theorem. Let $\mathbf{T}$ be a $\Sigma$-track algebra in dimension $n$ and consider a functor of $n$-graded categories

$$
K: \mathbb{Z}(\infty,-1)_{\otimes}^{n} \rightarrow \mathbf{T}
$$

which is a pre-chain complex (cf. \$4.1), and which is based on an a-resolution $A_{\bullet}$ of $X$ in $\mathbf{A}(c f .84 .3)$. Then there exists a functor

$$
K^{\prime}: \mathbb{Z}(\infty,-1)_{\otimes}^{n} \longrightarrow \mathbf{T}
$$

which coincides with $K$ in dimension $\leq n-1$, and which is an $n$-th order chain complex in $\mathbf{T}$ (based on $A_{\bullet}$ ).

The dual also holds.

13.3. Theorem. Let $\mathbf{T}$ be an $\Omega$-track algebra in dimension $n$ and consider a functor of $n$-graded categories

$$
L: \mathbb{Z}(+1,-\infty)_{\otimes}^{n} \longrightarrow \mathbf{T}
$$

which is a pre-chain complex and which is based on an $\mathbf{a - c o r e s o l u t i o n} A^{\bullet}$ of $Y$ in $\mathbf{A}$. Then there exists a functor

$$
L^{\prime}: \mathbb{Z}(+1,-\infty)_{\otimes}^{n} \longrightarrow \mathbf{T}
$$

which coincides with $L$ in dimension $\leq n-1$ and which is an $n$-th order chain complex in $\mathbf{T}$ (and is based on $A^{\bullet}$ ). 
Proof of Theorem 13.2. The functor $K^{\prime}$ is determined by $K$ in dimension $\leq n-1$ and by

$$
K^{\prime}\left(i, I_{n}\right)=K\left(i, I_{n}\right)+\alpha_{i}
$$

in dimension $n$. See Lemma 3.4. Here the elements $\alpha_{i}$ are obtained inductively as follows. We have to choose $\alpha_{i}$ for $i \geq n$ in such a way that the obstruction

$$
\xi\left(\alpha_{i-1}, \alpha_{i}\right)=\mathcal{O}_{B}\left(\delta_{i-n-1} K^{\prime}\left(i, I_{n}\right), b_{2}, \ldots, b_{n}, K^{\prime}\left(i-1, I_{n}\right) \delta_{i}\right)
$$

vanishes with $b_{r+1}=K\left(i, I_{r} \otimes I_{n-r}\right) \quad$ for $1 \leq r \leq n-1$, see 11.4. We start with $i=n+1$ and $\alpha_{n}:=0$. In this case $\left(\delta_{0}\right)_{*}$ is surjective since $A_{\bullet}$ is a resolution with $\delta_{0}: A_{0} \longrightarrow A_{-1}, A_{-1}=X$. The action rule implies that

$$
\xi\left(\alpha_{i-1}, \alpha_{i}\right)=\xi(0,0)+\varepsilon_{1}\left(\delta_{i-n-1}\right)_{*} \alpha_{i}+\varepsilon_{n+1}\left(\delta_{i}\right)^{*} \alpha_{i-1} .
$$

Here $\varepsilon_{1}, \ldots, \varepsilon_{n+1}$ are the orientation signs for the left cubical ball $B=T_{0}^{n}$. For $i=n+1$, we obtain for $\alpha_{n}=0$ the equation:

$$
\xi\left(0, \alpha_{n+1}\right)=\xi(0,0)+\varepsilon_{1}\left(\delta_{0}\right)_{*} \alpha_{n+1} .
$$

Since $\left(\delta_{0}\right)_{*}$ is surjective, there is an $\alpha_{n+1}$ with $\xi\left(0, \alpha_{n+1}\right)=0$.

We now consider (13.6) for $i=n+2$. Using Lemma 13.11, we shall show that

$$
\left(\delta_{0}\right)_{*} \xi\left(\alpha_{n+1}, \alpha_{n+2}\right)=0 .
$$

Since $A_{\bullet}$ is a resolution, this implies that

$$
\xi\left(\alpha_{n+1}, \alpha_{n+2}\right) \in \operatorname{Im}\left(\delta_{1}\right)_{*} .
$$

Since by (13.6) we have

$$
\xi\left(\alpha_{n+1}, \alpha_{n+2}\right)=\xi(0,0)+\varepsilon_{1}\left(\delta_{1}\right)_{*} \alpha_{n+2}+\varepsilon_{n+1} \delta_{n+2}^{*} \alpha_{n+1},
$$

we can choose $\alpha_{n+2}$ with $\xi\left(\alpha_{n+1}, \alpha_{n+2}\right)=0$. Thus we get by induction $\alpha_{i} \quad(i \geq n)$, such that $\xi\left(\alpha_{i-1}, \alpha_{i}\right)=0$. Hence $K^{\prime}$, defined by (13.4), satisfies the obstruction property, and hence is an $n$-th order chain complex as in the Theorem. In the next Lemma we show that (13.7) holds.

We introduce the following notation on the 'boundary' of $I_{n+1} \quad(n \geq 0)$. Let

$$
\partial I_{1}:=\emptyset \otimes \emptyset
$$

and for $n \geq 1$, let

$$
\partial I_{n+1}=\left(\emptyset \otimes I_{n}, I_{1} \otimes I_{n-1}, I_{2} \otimes I_{n-2}, \ldots, I_{n-1} \otimes I_{1}, I_{n} \otimes \emptyset\right) .
$$

(see (4.7)) .

Given a pre-chain complex $K^{\prime}: \mathbb{Z}(\infty,-1)_{\otimes}^{n} \longrightarrow \mathbf{T}$, we obtain for $i \geq n \geq 1$ the obstruction element

$$
\mathcal{O}_{B} K^{\prime}\left(i, \partial I_{n+1}\right)
$$

where $B=T_{0}^{n}$. This corresponds to (13.5) in the proof above.

13.11. Hauptlemma. Let $n \geq 1$ and $i \geq n+2$, and assume that

$$
\mathcal{O}_{B} K^{\prime}\left(i-1, \partial I_{n+1}\right)=0 \text {. }
$$

Then we also have

$$
\mathcal{O}_{B} K^{\prime}\left(i, \emptyset \otimes \partial I_{n+1}\right)=0
$$


13.12. Remark. For the proof of Hauptlemma 13.11, we use the following equation given by the triviality rule of $\oint 11.1(5)$ :

$$
\mathcal{O}_{B} K^{\prime}\left(i, \partial_{r, s}\right)=0
$$

for with $r+s=n+1, \quad r, s \geq 1$, and $i \geq n$, where

$$
\partial_{r, s}:=\left(\left(\partial I_{r}\right) \otimes I_{s}, I_{r} \otimes\left(\partial I_{s}\right)\right) .
$$

The hypothesis of the Lemma implies that

$$
\mathcal{O}_{B} K^{\prime}\left(i,\left(\partial I_{n+1}\right) \otimes \emptyset\right)=0
$$

by the naturality rule (11.2).

One should think of the Lemma as relating the obstructions associated to the boundaries of the various sub- $(n+1)$-balls of the 'corner' left cubical ball $T_{0}^{n+1} \subseteq \partial I^{n+2}$ (see 99.2). The two obstructions of the Lemma are those associated to the $(n+1)$-balls $\emptyset \otimes I_{n+1}$ and $I_{n+1} \otimes \emptyset$, respectively. However, we must describe them in terms of the lists of $n+1$ left $n$-cubes constituting their boundaries, namely,

$$
X:=\left(\emptyset \otimes \emptyset \otimes I_{n}, \emptyset \otimes \partial_{1, n}, \emptyset \otimes \partial_{2, n-1}, \ldots \emptyset \otimes \partial_{n, 1}, \emptyset \otimes I_{n} \otimes \emptyset\right)
$$

(cf. (13.10) $)$ and

$$
Y:=\left(\emptyset \otimes I_{n} \otimes \emptyset, \partial_{1, n} \otimes \emptyset, \partial_{2, n-1} \otimes \emptyset, \ldots \partial_{n, 1} \otimes \emptyset, I_{n} \otimes \emptyset \otimes \emptyset\right),
$$

respectively. The vanishing of the obstruction (13.14) means that $Y \sim 0$, and similarly $\mathcal{O}_{B} K^{\prime}\left(i, \emptyset \otimes \partial I_{n+1}\right) \quad$ vanishes if and only if $X \sim 0$.

All the other $(n+1)$-cubes of $T_{0}^{n+1}$ are non-trivial $\otimes$-products, with boundaries of the form $\partial_{r, s}$ for $r+s=n+1 \quad(r, s \geq 1)$, so the corresponding obstructions vanish by the triviality rule. This allows us to extend the $(n+1)$-balls bounded by $X$ and $Y$, respectively, to larger $(n+1)$-balls bounded by $X^{\prime}$ and $Y^{\prime}$, in such a way that $X^{\prime} \sim X$ and $Y^{\prime} \sim Y$ (using the complement rule of Lemma 10.6).

The Lemma is then proved by showing that one can choose these extensions so that $X^{\prime}=Y^{\prime} \quad$ (because we have two complementary sub- $(n+1)$-balls of $T_{0}^{n+1}$ with the same boundary). This implies that the corresponding obstructions are equal to each other, and thus both vanish by (13.14).

Proof of Hauptlemma 13.11 for $n=1$. In this case the triviality rule (13.13) yields:

$$
\partial_{1,1}:=\left(\emptyset \otimes \emptyset \otimes I_{1}, I_{1} \otimes \emptyset \otimes \emptyset\right) \sim 0
$$

while by (13.14) we have

$$
Y:=\left(\emptyset \otimes I_{1} \otimes \emptyset, I_{1} \otimes \emptyset \otimes \emptyset\right) \sim 0 .
$$

Thus by the complement rule and (13.17) we see that

$$
X:=\left(\emptyset \otimes \emptyset \otimes I_{1}, \emptyset \otimes I_{1} \otimes \emptyset\right)
$$

and

$$
X^{\prime}:=\left(I_{1} \otimes \emptyset \otimes \emptyset, \emptyset \otimes I_{1} \otimes \emptyset\right)
$$

satisfy $X \sim X^{\prime}=Y \sim 0$ by (13.18). 
Proof of Hauptlemma 13.11 for $n=2$. By (13.13), we find:

$$
\left(\emptyset \otimes I_{1} \otimes I_{1}, I_{1} \otimes \emptyset \otimes I_{1}, I_{2} \otimes \emptyset \otimes \emptyset\right) \sim 0
$$

and

$$
\left(\emptyset \otimes \emptyset \otimes I_{2}, I_{1} \otimes \emptyset \otimes I_{1}, I_{1} \otimes I_{1} \otimes \emptyset\right) \sim 0 .
$$

By the assumption (13.14) we have

$$
Y=\left(\emptyset \otimes I_{2} \otimes \emptyset, I_{1} \otimes I_{1} \otimes \emptyset, I_{2} \otimes \emptyset \otimes \emptyset\right) \sim 0 .
$$

We have to show

$$
X=\left(\emptyset \otimes \emptyset \otimes I_{2}, \emptyset \otimes I_{1} \otimes I_{1}, \emptyset \otimes I_{2} \otimes \emptyset\right) \sim 0 .
$$

By the complement rule and (13.20) (removing $\emptyset \otimes \emptyset \otimes I_{2}$ ) we find that

$$
X \sim X^{\prime}:=\left(I_{1} \otimes \emptyset \otimes I_{1}, I_{1} \otimes I_{1} \otimes \emptyset, \emptyset \otimes I_{1} \otimes I_{1}, \emptyset \otimes I_{2} \otimes \emptyset\right),
$$

and similarly by (13.19) (removing $\left.I_{2} \otimes \emptyset \otimes \emptyset\right)$ :

$$
Y \sim Y^{\prime}:=\left(\emptyset \otimes I_{2} \otimes \emptyset, I_{1} \otimes I_{1} \otimes \emptyset, \emptyset \otimes I_{1} \otimes I_{1}, I_{1} \otimes \emptyset \otimes I_{1}\right) .
$$

Thus $X \sim X^{\prime}=Y^{\prime} \sim Y \sim 0$.

Proof of Hauptlemma 13.11. By (13.13) we have the relations

$$
\partial_{r, s} \sim 0 \quad \text { for } r+s=n+1, r \geq 1 .
$$

By (13.14), the hypothesis of the Lemma implies that

$$
Y:=\left(\partial I_{n+1}\right) \otimes \emptyset \sim 0
$$

We have to show that

$$
X=\emptyset \otimes\left(\partial I_{n+1}\right) \sim 0 .
$$

We now define inductively a sequence of collections $Y^{(s)}$ of $n$-facets of $T_{0}^{n+1}$ for $0 \leq s \leq n$. We start with $Y^{(0)}:=Y$ as in (13.16), and define $Y^{(1)}$ by adding

$$
\partial_{n, 1}=\left(\emptyset \otimes I_{n-1} \otimes I_{1}, I_{1} \otimes I_{n-2} \otimes \otimes I_{1}, \ldots, I_{n-1} \otimes I_{1} \otimes I_{1}, I_{n} \otimes \emptyset \otimes \emptyset\right)
$$

and removing the common $n$-cube $I_{n} \otimes \emptyset \otimes \emptyset$ to obtain:

$$
Y^{(1)}=(\underbrace{\emptyset \otimes I_{n} \otimes \emptyset, \ldots, I_{n-1} \otimes I_{1} \otimes \emptyset}, \underbrace{\emptyset \otimes I_{n-1} \otimes I_{1}, \ldots, I_{n-1} \otimes I_{1} \otimes I_{1}}) .
$$

Note that $Y \sim Y^{(1)}$, since the associated obstructions are equal by the triviality and complement rules.

By induction we define $Y^{(s)}$ by adding $\partial_{n+1-s, s}$ to $Y^{(s-1)}$, so that

$$
\begin{gathered}
Y^{(s)}=(\underbrace{\emptyset \otimes I_{n} \otimes \emptyset, \ldots, I_{n-s} \otimes I_{s} \otimes \emptyset}, \underbrace{\emptyset \otimes I_{n-2} \otimes I_{2}, \ldots, I_{n-s} \otimes I_{s-2} \otimes I_{2}}, \ldots, \underbrace{\emptyset \otimes I_{n-1} \otimes I_{n-s} \otimes I_{s}, \ldots, \ldots, I_{n-s} \otimes I_{s-1} \otimes I_{n-s} \otimes \otimes I_{s}})
\end{gathered}
$$

(consisting of $s+1$ groups of $n-s+1 \quad n$-cubes each). Again by the complement rule $Y^{(s)} \sim Y^{(s-1)}$, so by induction $Y^{(s)} \sim Y \sim 0$. Moreover, since the $n$-cube facets of $T_{0}^{n+1}$ common to $Y^{(s-1)}$ and

$$
\partial_{s, n+1-s}=\left(\left(\partial I_{s}\right) \otimes I_{n+1-s}, I_{s} \otimes\left(\partial I_{n+1-s}\right)\right)
$$


form the left cubical $n$-ball $I_{s} \otimes\left(\partial I_{n+1-s}\right)$, by induction we see also that $Y^{(s)}$ is the boundary of an $n$-ball (the connected sum of $\partial_{s, n+1-s}$ and $Y^{(s-1)}$ ).

Similarly, if we start with $X^{(0)}:=X$ as in (13.15), and define $X^{(1)}$ by adding

$$
\partial_{1, n}=(\emptyset \otimes \emptyset \otimes I_{n}, \underbrace{I_{1} \otimes \emptyset \otimes I_{n-1}, \ldots, I_{1} \otimes I_{n-2} \otimes I_{1}, I_{1} I_{n-1} \otimes \emptyset})
$$

and removing the common $n$-ball $\emptyset \otimes \emptyset \otimes I_{n}$, we obtain

$$
X^{(1)}=(\underbrace{\emptyset \otimes I_{1} \otimes I_{n}, \ldots, \emptyset \otimes I_{n} \otimes \emptyset}, \underbrace{I_{1} \otimes \emptyset \otimes I_{n-1}, \ldots, I_{1} \otimes I_{n-1} \otimes \emptyset}) .
$$

By induction define $X^{(r)}$ by adding $\partial_{r, n+1-r}$ to $X^{(r-1)}$, so that

$$
\begin{gathered}
X^{(r)}=(\underbrace{\emptyset \otimes I_{r} \otimes I_{n-r}, \ldots, \emptyset \otimes I_{n} \otimes \emptyset}, \underbrace{\underbrace{I_{1} \otimes I_{r-1} \otimes I_{n-r}, \ldots, I_{1} \otimes I_{n-1} \otimes \emptyset}_{1}}, \\
\underbrace{I_{2} \otimes I_{r-2} \otimes I_{n-r}, \ldots, I_{2} \otimes I_{n-2} \otimes \emptyset}, \ldots, \underbrace{I_{r} \otimes \emptyset \otimes I_{n-r}, \ldots, I_{r} \otimes I_{n-r} \otimes \emptyset})
\end{gathered}
$$

Again $X^{(r)}$ is the boundary of an $n$-ball, and by the complement rule $X^{(r)} \sim X^{(r-1)}$, so by induction $X^{(r)} \sim X$.

Finally, assume $r+s=n \quad$ (e.g., for $s=\left\lfloor\frac{n}{2}\right\rfloor$ and $r=\left\lceil\frac{n}{2}\right\rceil$ ). In this case, by direct inspection we see that the $(r+1) \cdot(n-r+1) n$-cubes of (13.27) are just a reordering of the $(s+1) \cdot(n-s+1) n$-cubes of (13.26) $)$, so $X^{(r)}=Y^{(s)}$. The reason this happens is that the $(n+1)$-cube facets of $T_{0}^{n+1}$ forming the interiors of the two $(n+1)$-balls bounded by $X^{(r)}$ and $Y^{(s)}$, respectively, constitute a partition of all the $(n+1)$-cubes, so they partition the $(n+1)$-ball $T_{0}^{n+1}$ into two complementary $(n+1)$-balls with a common boundary $X^{(r)}=Y^{(s)}$.

Thus

$$
X \sim X^{(r)}=Y^{(s)} \sim Y \sim 0
$$

so the obstruction $\mathcal{O}_{B} K^{\prime}\left(i, \emptyset \otimes \partial I_{n+1}\right)$ associated to $X$ vanishes, as claimed.

\section{Algebras of left cubical balls and the proof of the Resolution THEOREM}

In order to prove Resolution Theorem 8.2, we need to relate algebras of left cubical $n$-balls for different $n$, as follows:

14.1. Definition. A total algebra of left $n$-cubical balls $\mathbf{T}(\leq n)$ is a sequence of algebras of left $m$-cubical balls

$$
\mathbf{T}(m)=\left(\mathbf{T}(m), \mathbf{A}, D_{m}, \mathcal{O}^{m}\right)
$$

for $m=1,2, \ldots, n$, together with quotient functor

$$
q: \mathbf{T}(m+1)^{m} \rightarrow \mathbf{T}(m)
$$

which is the identity on objects, is full, and is the identity functor on $(m-1)$-skeleta:

$$
q\left(\mathbf{T}(m+1)^{m-1}\right)=\mathbf{T}(m)^{m-1} .
$$

Moreover, the boundary property of Lemma (10.5) holds - that is, for $B=T_{0}^{m}$, we have

$$
\mathcal{O}_{B}^{m}\left(a_{1}, \ldots, a_{m+1}\right)=0
$$

if and only if there exists $\bar{a} \in \mathbf{T}(m+1)_{m+1} \quad$ with $q\left(\partial^{i} \bar{a}\right)$ representing $a_{i}$ for $i=1, \ldots, m+1$. 
14.2. Example. Let $\mathbf{C}$ be a category enriched in pointed spaces with zero maps. Then

$$
\mathrm{Nul}_{\leq n} \mathbf{C}:=\left(\mathrm{Nul}_{n} \mathbf{C}, \mathrm{Nul}_{n-1} \mathbf{C}, \ldots, \mathrm{Nul}_{1} \mathbf{C}\right)
$$

is a total algebra of left $n$-cubical balls.

14.3. Definition. We say that $\mathbf{T}(\leq n)$ is a $\Sigma$-algebra of left cubical balls if $\mathbf{A}=$ $(\mathbf{A}, \mathbf{a}, \Sigma)$ is a $\Sigma$-algebra as in Definition 6.4 and

$$
D_{m}(X, Y)=\operatorname{Hom}_{\mathbf{A}}\left(\Sigma^{m} X, Y\right)
$$

for $m=1, \ldots, n$ and $X$ in $\mathbf{a}$ and $Y$ in $\mathbf{A}$.

Dually we say $\mathbf{T}(\leq n)$ is an $\Omega$-algebra of left cubical balls if $\mathbf{A}=(\mathbf{A}, \mathbf{a}, \Omega)$ is an $\Omega$-algebra as in (6.5) and

$$
D_{m}(X, Y)=\operatorname{Hom}_{\mathbf{A}}\left(X, \Omega^{m} Y\right)
$$

for $m=1, \ldots, n$ and $X$ in $\mathbf{A}$ and $Y$ in $\mathbf{a}$.

14.4. Example. If $\mathbf{C}$ is a $\Sigma$-mapping algebra, then $\mathrm{Nul}_{\leq n} \mathbf{C}$ is a $\Sigma$-algebra of left cubical balls. If $\mathbf{C}$ is an $\Omega$-mapping algebra, then $\mathrm{Nul}_{\leq n} \mathbf{C}$ is an $\Omega$-algebra of left cubical balls.

The following Resolution Theorems now generalize those of Section 8

14.5. Theorem. Let $\mathbf{T}(\leq n)$ be a $\Sigma$-algebra of left cubical balls and let $A_{\bullet}$ be an a-resolution of $X$ in $\mathbf{A}$. Then there exists an $n$-th order chain complex

$$
K: \mathbb{Z}(\infty,-1)_{\otimes}^{n} \longrightarrow \mathbf{T}(n)
$$

which is based on $A_{\bullet}$. We call $K$ an $n$-th order resolution of $X$ in $\mathbf{T}(n)$.

14.6. Theorem. Let $\mathbf{T}(\leq n)$ be an $\Omega$-algebra of left cubical balls and let $A^{\bullet}$ be an $\mathbf{a}$-coresolution of $Y$ in $\mathbf{A}$. Then there exists an $n$-th order chain complex

$$
L: \mathbb{Z}(+1,-\infty)_{\otimes}^{n} \rightarrow \mathbf{T}(n)
$$

which is based on $A^{\bullet}$. We call $L$ an $n$-th order coresolution of $Y$ in $\mathbf{T}(n)$.

Proof. The boundary property shows that there exists a functor

$$
K^{\prime}(1): \mathbb{Z}(\infty,-1)_{\otimes}^{1} \longrightarrow \mathbf{T}(1)
$$

which satisfies the inclusion property and which is based on $A_{\bullet}$. Hence by Theorem 13.2 we have a first-order chain complex $K(1)$ which is based on $A_{\bullet}$. Now the boundary property shows that there is a functor

$$
K^{\prime}(2): \mathbb{Z}(\infty,-1)_{\otimes}^{1} \longrightarrow \mathbf{T}(2)
$$

which satisfies the inclusion property and which based on $A_{\bullet}$. Again the boundary property shows there exists $K^{\prime}(3)$, so that by Theorem 13.2 one obtains $K(3)$. Inductively, we thus have $K=K(n)$.

14.7. Example. Let $\mathbf{C}$ be the Eilenberg-Mac Lane $\Omega$-mapping algebra. Then minimal coresolutions $A^{\bullet}$ of $Y$ are defined in $\mathbf{A}$ and hence we can find an $n$-th order coresolution of $Y$ in $\operatorname{Nul}_{\leq n} \mathbf{C}$ based on $A^{\bullet}$. We call $\mathrm{Nul}_{\leq n} \mathbf{C}$ the algebra of cohomology operations of order $\leq n+1$. This is an $\Omega$-algebra of left cubical balls. It is convenient to consider the dual of $\mathrm{Nul}_{\leq n} \mathbf{C}$, which is a $\Sigma$-algebra of left cubical balls and for which $\mathbf{a}$ is the category of finitely generated free modules over the Steenrod algebra. 
14.8. Remark. The main result of [B2] computes the algebra of cohomology operations of order $\leq 2$ in terms of a bigraded differential algebra $\mathcal{B}$ over the ring $\mathbb{Z} / p^{2}$. This leads to the conjecture that the algebra of cohomology operations of order $\leq n$ $(n \geq 1)$, can also be described up to equivalence by a bigraded differential algebra over $\mathbb{Z} / p^{2}$.

\section{HighER ORDER Ext-GROUPS}

In this section we deduce from higher order resolutions the associated higher order derived functors, which are higher order Ext-groups. We shall see that the $E_{n}$-term in the Adams spectral sequence is such a higher order Ext-group for $n \geq 3$.

It is classical that the $E_{2}$ of the Adams spectral sequence is given by the 'primary' Ext-groups of homological algebra, see (6.5). In [BJ2] we studied the secondary Ext-groups which determine $E_{3}$.

15.1. Definition. Let $\mathbf{T}(\leq n) \quad(n \geq 1) \quad$ be a $\Sigma$-algebra of left cubical balls, so for each $m=1, \ldots, n$ we have the algebra of left $m$-cubical balls

$$
\mathbf{T}(m)=\left(\mathbf{T}(m), \mathbf{A}, D_{m}, \mathcal{O}^{m}\right)
$$

with $\mathbf{a} \subset \mathbf{A}$ and $D_{m}(A, X)=\operatorname{Hom}_{\mathbf{A}}\left(\sum^{m} A, X\right)$ for objects $A$ in a and $X$ in $\mathbf{A}$. Let $A_{\bullet}$ be an a-resolution of $X$ in $\mathbf{A}$ and let

$$
K: \mathbb{Z}(\infty,-1)_{\otimes}^{n} \longrightarrow \mathbf{T}(n)
$$

be an $n$-th order resolution of $X$ based on $A_{\bullet}$ (see Theorem 14.5). Furthermore, let $Y$ be another object of $\mathbf{A}$, and consider the diagram in $\mathbf{A}$ :

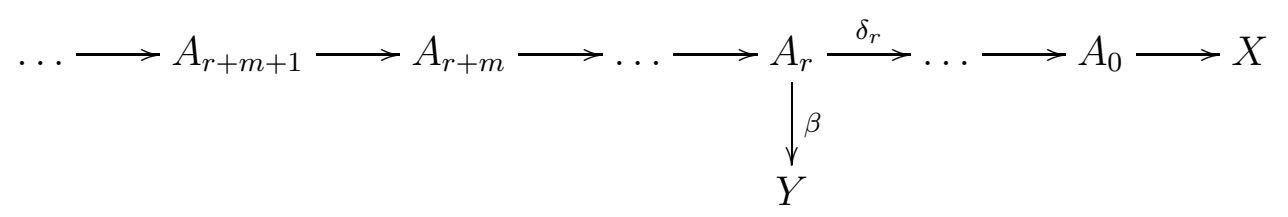

The row of the diagram is the a-resolution $A$. of $X$. We assume that $\beta$ is a cocycle, that is,

$$
\beta \delta_{r+1}=0
$$

Then $\beta$ represents an element $\{\beta\}$ in the Ext-group

$$
\begin{aligned}
E_{2}^{r, 0} & =\operatorname{Ext}_{\mathbf{A}}^{r}(X, Y) \\
& =\operatorname{H}^{r} \operatorname{Hom}_{\mathbf{A}}\left(A_{\bullet}, Y\right) \\
& =\operatorname{Ker} \delta_{r+1}^{*} / \operatorname{Im} \delta_{r}^{*},
\end{aligned}
$$

where

$$
\delta_{r}^{*}: \operatorname{Hom}_{\mathbf{A}}\left(A_{r-1}, Y\right) \longrightarrow \operatorname{Hom}_{\mathbf{A}}\left(A_{r}, Y\right) .
$$

Using the a-resolution $\Sigma^{s} A$. of $\Sigma^{s} X$, we get accordingly for $s \geq 0$ the bigraded Ext-group (see (6.5) ),

$$
E_{2}^{r, s}=\operatorname{Ext}_{\mathbf{A}}^{r}\left(\Sigma^{s} X, Y\right)
$$

In Definition 15.8 below we shall define a differential

$$
d_{2}=d_{2}^{r, s}: E_{2}^{r, s} \rightarrow E_{2}^{r+2, s+1} .
$$


Moreover, inductively for $m \geq 2$ we consider subquotients $E_{m}^{r, s}$ of $E_{2}^{r, s}$, together with differentials

$$
d_{m}=d_{m}^{r, s}: E_{m}^{r, s} \longrightarrow E_{m}^{r+m, s+m-1}
$$

satisfying $d_{m} d_{m}=0$, and

$$
E_{m+1}^{r, s}=\operatorname{Ker}\left(d_{m}^{r, s}\right) / \operatorname{Im}\left(d_{m}^{r-m, s-m+1}\right) .
$$

We call $E_{m}^{r, 0}$ for $m=2, \ldots, n+1$ the higher order Ext-groups associated to the $n$-th order resolution $K$ of $X$ above. Replacing $X$ by $\Sigma^{s} X$, we obtain the groups $E_{m}^{r, s}$, accordingly.

15.8. Definition. Let $\bar{\beta} \in E_{m+1}^{r, 0}$ be represented by $\{\beta\} \in E_{2}^{r, 0} \quad(1 \leq m \leq n)$, and let $L$ be an $(m-1)$-order chain complex

$$
L: \mathbb{Z}(\infty, r-1)_{\otimes}^{m-1} \longrightarrow \mathbf{T}(m-1)
$$

based on the diagram

$$
\ldots \rightarrow A_{r+m+1} \ldots \rightarrow A_{r+1} \rightarrow A_{r} \stackrel{\beta}{\rightarrow} Y,
$$

in A. Such an $L$ exists precisely because $\beta$ survives to the $E_{m+1}$-term. We assume also that $L$ restricted to $\mathbb{Z}(\infty, r)$ coincides with the $(m-1)$-skeleton of $K$ in $\mathbf{T}(m-1)$. The boundary property in $\mathbf{T}(m)$ shows that there is a functor

$$
\hat{L}: \mathbb{Z}(\infty, r-1)_{\otimes}^{m} \longrightarrow \mathbf{T}(m)
$$

which is a pre-chain complex such that $\hat{L}$ restricted to $\mathbb{Z}(\infty, r)$ coincides with the $m$-skeleton of $K$, and such that the $(m-1)$-skeleton $\hat{L}(m-1)$ of $\hat{L}$ satisfies $q \hat{L}(m-1)=L \quad$ in $\mathbf{T}(m-1)$. We then obtain the obstruction

$$
\mathcal{O} \hat{L}\left(r+m+1, \partial I_{m+1}\right)=\mathcal{O}_{B}\left(b_{1}, \ldots, b_{m+1}\right) \in \operatorname{Hom}_{\mathbf{A}}\left(\Sigma^{m} A_{r+m+1}, Y\right),
$$

where $B=T_{0}^{m}$ and

$$
\hat{L}\left(r+m+1, \partial I_{m+1}\right)=\left\{\begin{array}{l}
b_{1}=\hat{L}\left(r+m+1, \emptyset \otimes I_{m}\right), \\
b_{k+1}=\hat{L}\left(r+m+1, I_{k} \otimes I_{m-1}\right), \quad 1 \leq k \leq m-1, \\
b_{m+1}=\hat{L}\left(r+m+1, I_{m} \otimes \emptyset\right)
\end{array}\right.
$$

(see (4.7)). The element $\mathcal{O} \hat{L}\left(r+m+1, \partial I_{m+1}\right)$ represents the value of the differential

$$
d_{m+1}^{r, 0}(\bar{\beta}) \in E_{m+1}^{r+m+1, m} .
$$

15.9. Theorem. Let $\mathbf{T}(\leq n)=\mathrm{Nul}_{\leq n} \mathbf{C}$ be the $\Sigma$-algebra of left cubical balls given by the complete $\Sigma$-mapping algebra $\mathbf{C}$ of Example 7.3. Then Definition 15.8 yields a well defined sequence of Ext-groups $E_{m}^{r, s}$ for $m=2, \ldots, n+2$. These groups depend on the weak equivalence class of the $\Sigma$-algebra of left cubical balls $\mathbf{T}(\leq n)$, and not on the choice of the $n$-th order resolution of $X$.

15.10. Remark. Some additional assumptions on a $\Sigma$-algebra of left cubical balls $\mathbf{T}(\leq$ $n)$ are needed in Theorem 15.9 in order to guarantee that the last differential $d_{n+2}: E_{n+2} \rightarrow E_{n+2} \quad$ still satisfies $d_{n+2} \circ d_{n+2}=0$. The requisite assumptions are axiomatized in the Appendix, but they are certainly satisfied in Example 7.3 (or any other case where we actually have a spectral sequence with these differentials). 
15.11. Theorem. Let $\mathbf{T}(\leq n)=\mathrm{Nul}_{\leq n} \mathbf{C}$ be the $\Sigma$-algebra of left cubical balls given by the dual $\mathbf{C}$ of the Eilenberg-Mac Lane $\Omega$-algebra of left cubical balls. Then the Extgroups $E_{m}^{r, s} \quad(2 \leq m \leq n+2)$, yield the $m$-term $E_{m}$ of the Adams spectral sequence which converges to the stable homotopy set $\{Y, X\}$ for finite spectra $X$ and $Y$.

For $n=1$ this result is proved in [BJ2, Section 7]. Theorems 15.9 and 15.11 are proved for all $n \geq 1$ in the following Section.

\section{Strictification of Higher ORDER RESOLUtions}

In this section we show that any higher order resolution is actually equivalent to a strict resolution, which we call its strictification. This is done by induction: the main difficulty is choosing the correct induction hypotheses. Otherwise, the proof uses a long chain of standard arguments in Quillen model categories.

It is well-known how to derive from such a strict resolution the corresponding (Adams) spectral sequence, thus implying Theorems [15.9] and 15.11.

Throughout this section $\mathbf{C}$ will be a $\Sigma$-mapping algebra of the type described in Example 7.3, so the reader may take $\mathbf{C}$ to be $\mathbf{S p e c}$.

16.1. Notation. For such a $\mathbf{C}$, we have the additive category $\mathbf{A}=\pi_{0} \mathbf{C}$, and the full additive subcategory $\mathbf{a}=\pi_{0}\{\mathcal{X}\}$ given by the class of spectra $\mathcal{X}$ in 7.3 , Let $\widehat{\mathbf{a}}$ denote the full subcategory of $\mathbf{A}$ consisting of all objects $A$ in which are isomorphic in $\mathbf{A}$ to an object in a, with

$$
\mathbf{a} \subseteq \widehat{\mathbf{a}} \subseteq \mathbf{A}
$$

16.3. Definition. Let $\mathbf{T}$ be an $n$-graded category (such as $\operatorname{Nul}_{n} \mathbf{C}$ or $\operatorname{nul}_{n} \mathbf{C}$ ) with a quotient functor $q: \mathbf{T}^{0} \rightarrow \mathbf{A}$. Let $K, L: \mathbb{Z}(\infty,-1)_{\otimes}^{n} \rightarrow \mathbf{T}$ be functors of $n$-graded categories. A weak equivalence $\tau: K \rightarrow L$ over an object $X \in \mathbf{T}^{0}$ is a natural transformation $\tau$ which for objects $i$ in $\mathbb{Z}_{\otimes}$ consists of a map

$$
\tau_{i}: K_{i} \rightarrow L_{i} \text { in } \mathbf{T}^{0}
$$

which induces an isomorphism $q \tau_{i}$ in $\mathbf{A}$. For $i=-1$, the map $\tau_{-1}: K_{-1}=X=L_{-1}$ is the identity of $X$. For a morphism $V: i \rightarrow j$ in $\mathbb{Z}_{\otimes}$, we have the commutative diagram in $\mathbf{T}$ :

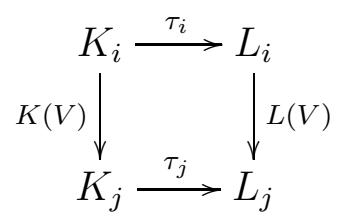

or equivalently, $\tau_{j} K(V)=L(V) \tau_{i}$. Let $\sim$ be the equivalence relation generated by weak equivalences over $X$.

16.4. Lemma. Let $K, L: \mathbb{Z}(\infty,-1)_{\otimes}^{n} \rightarrow \mathrm{Nul}_{n} \mathbf{C}$ be $n$-th order resolutions of $X$. If $K \sim L$ are weakly equivalent over $X$, then the higher Ext-groups defined by $K$ and $L$ are isomorphic.

We shall show that the higher Ext-groups actually do not depend on the choice of resolution of $X$. For this, we use the strictification of resolutions. 
16.5. Definition. Let $K: \mathbb{Z}(\infty,-1)_{\otimes}^{n} \rightarrow \mathbf{T}$ be a functor of $n$-graded categories, and $N \geq 0$. We say that $K$ is $N$-strict if for all $i \leq N$ and $k=1, \ldots, n$ we have $K\left(i, I_{k}\right)=o$. In this case $\delta_{i}=K(i, \emptyset): K_{i} \rightarrow K_{i-1} \quad$ yields a sequence of maps

$$
K_{N} \stackrel{\delta}{\rightarrow} K_{N-1} \stackrel{\delta}{\rightarrow} \ldots \stackrel{\delta}{\rightarrow} K_{0} \stackrel{\delta}{\rightarrow} K_{-1}
$$

in $\mathbf{T}^{0}$, with $K_{-1}=X$ and $\delta \delta=o$.

When $\mathbf{T}$ is $\mathrm{Nul}_{n} \mathbf{C}$ or $\operatorname{nul}_{n} \mathbf{C}$ for a topologically enriched model category $\mathbf{C}$ as above, we say that $K$ as above is $N$-fibrant if there are fiber sequences

$$
Z_{i} \stackrel{j_{i}}{\rightarrow} K_{i} \stackrel{p_{i}}{\rightarrow} Z_{i-1} \quad p_{i} \circ j_{i}=o,
$$

in the model category $\mathbf{C}$ with $\delta_{i}=j_{i-1} p_{i}$ for each $0 \leq i<N$, and $\delta_{N}$ admits a factorization

$$
K_{N} \stackrel{\bar{p}_{N}}{\longrightarrow} Z_{N-1} \stackrel{j_{N-1}}{\longrightarrow} K_{N-1}
$$

Note that $p_{i}: K_{i} \rightarrow Z_{i-1}$ above is a fibration, but $\bar{p}_{N}: K_{N} \rightarrow Z_{N-1}$, need not be a fibration. More generally, throughout this section we use $p$ for fibrations, while $\bar{p}$ need not be a fibration.

Moreover, $K$ is $N$-exact if for each $0 \leq i<N$ and $A$ in a, the induced sequence

$$
\operatorname{Hom}_{\mathbf{A}}\left(A, Z_{i}\right) \rightarrow \operatorname{Hom}_{\mathbf{A}}\left(A, K_{i}\right) \rightarrow \operatorname{Hom}_{\mathbf{A}}\left(A, Z_{i-1}\right)
$$

is a short exact sequence of abelian groups.

16.7. Theorem. Let $n \geq 1$ and $N \geq 0$, and let $K: \mathbb{Z}(\infty,-1)_{\otimes}^{n} \rightarrow \operatorname{Nul}_{n} \mathbf{C}$ be an $n$-th order resolution of $X$ based on the $\mathbf{a}$-resolution $A_{\bullet}$ of $X$ in $\mathbf{A}$. Then there exists an $N$-strict $N$-fibrant $N$-exact $n$-th order resolution $L$ of $X$ based on an $\widehat{\mathbf{a}}$-resolution $\widehat{A}_{\bullet}$ of $X$ in A such that $L \sim K$ are weakly equivalent over $X$.

16.8. Remark. The dual of Theorem 16.7 holds for coresolutions.

16.9. Notation. Here we use the bigger category $\widehat{\mathbf{a}}$ of $\$ 16.1$. The resolutions $A_{\bullet}$ and $\widehat{A}$. yield by the weak equivalence $L \sim K$ over $X$ the commutative diagram in A:

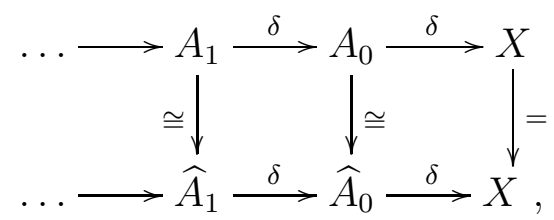

where the vertical arrows are isomorphisms in $\mathbf{A}$ and we have $A_{i}=K_{i}$ and $\widehat{A}_{i}=L_{i}$ for $i \geq-1$.

A $\Sigma$-mapping algebra $\mathbf{C}$ is given by an underlying model category and cubes

$$
I^{n} \longrightarrow \operatorname{Mor}_{\mathbf{C}}(X, Y) \text {, }
$$

having an adjoint

$$
\left(I^{n} \times X\right) /\left(I^{n} \times *\right) \longrightarrow Y
$$

where $(I \times X) /(I \times *)$ is the pointed cylinder on $X$.

16.11. Lemma. Theorem 16.7 holds for $n=1$.

Proof. By induction on $m \geq 0$ we construct a sequence of first-order resolutions $L^{(m)}$ of $X$ based on $A_{\bullet}$, such that: 
(i) $L^{(0)}$ is the original first-order resolution $K$.

(ii) For each $m \geq 1, \quad L^{(m)}$ is $m$-strict, $m$-fibrant, $m$-exact and weakly equivalent over $X$ to $L^{(m-1)} \quad$ (and thus by induction to $K$ ).

(iii) For each $m \geq 0, \quad L_{i}^{(m+1)}=L_{i}^{(m)}$ for all $i \neq m$ (so each new approximation differs from the previous one in a single dimension).

Assuming by induction that for some $N \geq 0$, we have such a $N$-strict, $N$-fibrant, and $n$-exact $K^{\prime}:=L^{(N)}$, we construct $L^{\prime}:=L^{(N+1)}$ as follows:

By assumption, we have a factorization of $\delta_{N-1}: K_{N-1}^{\prime} \rightarrow K_{N-2}^{\prime}$ as

$$
K_{N-1}^{\prime} \stackrel{p_{N-1}}{\rightarrow} Z_{N-2} \stackrel{j_{N-2}}{\longrightarrow} K_{N-2}^{\prime}
$$

with $Z_{N-1}$ the fiber of the fibration $p_{N-1}$ (and thus the kernel of $\delta_{N-1}$, since $j_{N-2}$ is monic). Because $K^{\prime}$ is $N$-strict, $\delta_{N-1} \circ \delta_{N}=o$, so $\delta_{N}: K_{N}^{\prime} \rightarrow K_{N-1}^{\prime}$ factors through $Z_{N-1}=\operatorname{Ker} \delta_{N-1}$ as

$$
K_{N}^{\prime} \stackrel{\bar{p}_{N}}{\longrightarrow} Z_{N-1} \stackrel{j_{N-1}}{\longrightarrow} K_{N-1}^{\prime}
$$

and in the model category $\mathbf{C}$ we may factor $\bar{p}_{N}$ as

$$
K_{N}^{\prime} \stackrel{i_{N}}{\longrightarrow} L_{N}^{\prime} \stackrel{p_{N}}{\longrightarrow} Z_{N-1}
$$

where $p_{N}$ is a fibration and $i_{N}$ is a trivial cofibration.

Hence we have a commuting diagram

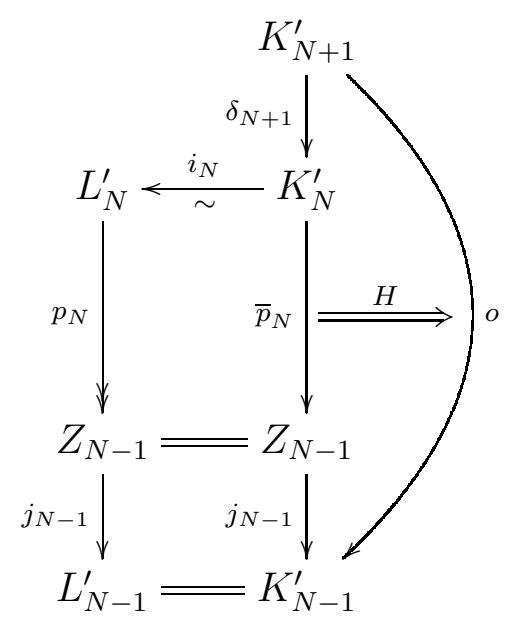

where we set $L_{i}^{\prime}:=K_{i}^{\prime}$ for all $i<N$, and $L_{N}^{\prime}$ is defined by (16.12). In fact, we shall also set $L_{i}^{\prime}:=K_{i}^{\prime}$ for all $i>N$, by (iii), so $L^{\prime}$ differs from $K^{\prime}$ only at the $N$-th slot.

Since $\delta_{N-1} \circ \delta_{N}=o, \delta_{N-1}=j_{N-2} \circ p_{N-1}$, and $j_{N-2}$ is monic, in fact $p_{N-1} \circ \delta_{N}=o$, so $p_{N-1} H$ is a self-nullhomotopy $o \Rightarrow o: K_{N+1}^{\prime} \rightarrow Z_{N-2}$, and thus induces a map $\alpha: \Sigma K_{N+1}^{\prime} \rightarrow Z_{N-2}$, with $j_{N-2} \alpha: \Sigma K_{N+1}^{\prime} \rightarrow K_{N-2}^{\prime}$ being (the adjoint of) the obstruction element $\mathcal{O} K^{\prime}\left(N+1, \partial I_{2}\right)$ of $\$ 4.5$ (for $n=1$ ), so it is nullhomotopic. Therefore, by (16.6) in the definition of $N$-exactness, $\alpha$ itself has a nullhomotopy $G: \alpha \Rightarrow o: \Sigma K_{N+1}^{\prime} \rightarrow Z_{N-2}$.

Expressing $G$ and $H$ in terms of maps out of products with cubes (or cubical horns) we obtain the solid commutative diagram of Figure 4, in which $H: \delta_{N} \circ \delta_{N+1} \Rightarrow o$ is extended from the left vertical map in the upper horn by constant homotopies along the 
horizontal edges of the square (with $\operatorname{Id}_{f}$ abbreviated to $f$ ). When the resulting map is post-composed with the fibration $p_{N-1}$, we can fill in the square by the homotopy of homotopies $G: \alpha \Rightarrow o$, with the maps $K_{N+1}^{\prime} \times I \rightarrow Z_{N-1}$ indicated along the edges of the square.

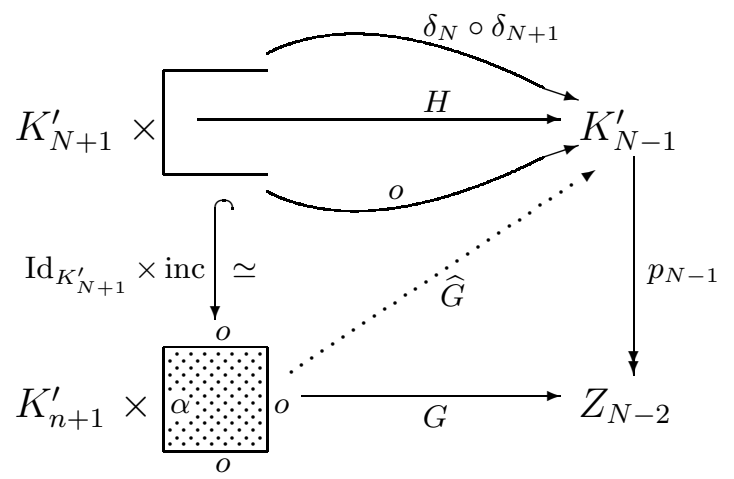

Figure 4. Lifting nullhomotopies

Since the left vertical inclusion is a trivial cofibration, we can use the lifting property in the model category $\mathbf{C}$ to obtain $\widehat{G}$, whose restriction to the right vertical edge of the lower square yields a new nullhomotopy $H^{\prime}: K_{N+1}^{\prime} \rightarrow K_{N-1}^{\prime}$ of $\delta_{N} \circ \delta_{N+1}$, which is homotopic to $H$ and thus represents the same track. Since $p_{N-1} H^{\prime}=\operatorname{Id}_{o}, \quad H^{\prime}$ factors through the kernel $Z_{N-1}$ of $p_{N-1}$ as $H^{\prime}=j_{N-1} \bar{H}$.

We then obtain the following diagram, where $I K_{N+1}^{\prime}$ is the cylinder, with inclusions $i_{0} \quad$ and $i_{1} \quad$ (setting $\left.L_{N+1}^{\prime}:=K_{N+1}^{\prime}\right)$.

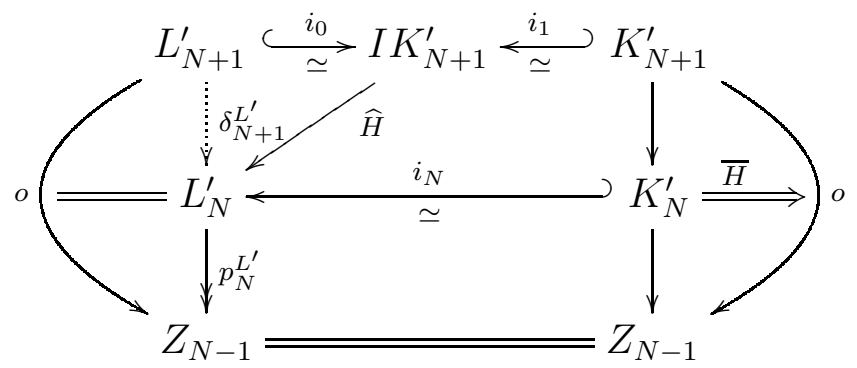

Here $\widehat{H}$ is a lift of $\bar{H}$ through $p_{N}$, so that the diagram commutes with $p_{N}^{L^{\prime}} \widehat{H} i_{0}=o$. Hence for $\delta_{N+1}^{L^{\prime}}:=\widehat{H} i_{0}$, the left hand side is $(N+1)$-strict and $(N+1)$-fibrant. Moreover, the left hand side is $(N+1)$-exact, that is:

$$
\left(p_{N}^{L^{\prime}}\right)_{*}=\operatorname{Hom}_{\mathbf{A}}\left(A, p_{N}\right): \operatorname{Hom}_{\mathbf{A}}\left(A, L_{N}^{\prime}\right) \rightarrow \operatorname{Hom}_{\mathbf{A}}\left(A, Z_{N-1}\right)
$$

is surjective for all $A$ in $\mathbf{a}$.

To show this, given $\alpha \in \operatorname{Hom}_{\mathbf{A}}\left(A, Z_{N-1}\right)$ we have $\left(\delta_{N-1} j_{N-1}\right)_{*} \alpha=0$, so that by exactness of $A_{\bullet}$ we have

$$
\left(j_{N-1}\right)_{*} \alpha=\left(\delta_{N}^{L^{\prime}}\right)_{*} \beta=\left(j_{N-1} p_{N}^{L^{\prime}}\right)_{*} \beta
$$

for some $\beta \in \operatorname{Hom}_{\mathbf{A}}\left(A, L_{N}^{\prime}\right)$. Thus $\alpha=\left(p_{N}^{L^{\prime}}\right)_{*} \beta$ by injectivity of $\left(j_{N-1}\right)_{*}$.

The fiber sequence for $p_{N}^{L^{\prime}}$ thus has $\operatorname{Hom}_{\mathbf{A}}\left(A, \Omega p_{N}\right)=\operatorname{Hom}_{\mathbf{A}}\left(\Sigma A, p_{N}\right)$ surjective, since $\Sigma A \in \mathbf{a}$, which implies that $\left(j_{N}\right)_{*}$ is one-to-one. 
We now construct weak equivalences

$$
L^{\prime} \stackrel{i_{0}}{\rightarrow} R \stackrel{i_{1}}{\longleftarrow} K^{\prime}
$$

where $i_{0}$ and $i_{1}$ are the identity in degrees $<N$. In degree $N$ the resolution $L^{\prime}$ is given by the diagram above. In dimension 0, diagram (16.16) is given by the commutative diagram:

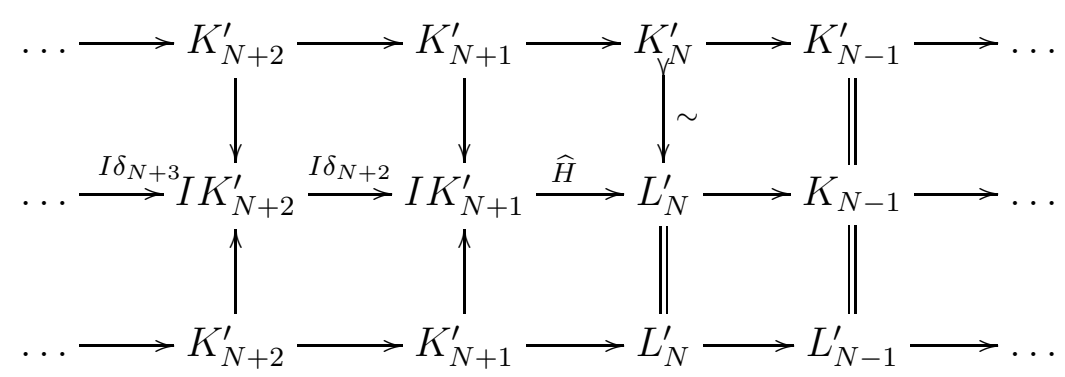

It is easy to find appropriate $R\left(N+1, I_{1}\right), R\left(N+2, I_{1}\right)$, and $R\left(M, I_{1}\right)=I K^{\prime}\left(M, I_{1}\right)$ for $M \geq N+3$, so that (16.16) is well defined. Here we use the adjoint maps in (16.10). This completes the proof of Theorem 16.7 for $n=1$.

16.18. Transport Lemma. Let $K$ be an $n$-th order resolution of $X$ in $\mathrm{Nul}_{n} \mathbf{C}$ with $(n-1)$-skeleton $K^{(n-1)}$. Let

$$
L^{(n-1)} \stackrel{f}{\rightarrow} K^{(n-1)} \stackrel{g}{\rightarrow} R^{(n-1)} \quad \text { in } \mathrm{Nul}_{n-1} \mathbf{C}
$$

be weak equivalences over $X$. Then there exist unique $n$-th order resolutions $L$ and $R$ in $\mathrm{Nul}_{n} \mathbf{C}$ together with weak equivalences

$$
L \stackrel{\tilde{f}}{\rightarrow} K \stackrel{\tilde{g}}{\rightarrow} R
$$

which, restricted to $(n-1)$-skeleta, coincide with $f$ and $g$ respectively.

Proof. Given a map $u: \partial I^{n} \rightarrow U$ in Top, let $\left[I^{n}, U\right]^{u}$ denote the set of homotopy classes of extensions of $u$ along the inclusion $\partial I^{n} \hookrightarrow I^{n} \quad$ (relative to $\partial I^{n}$ ). By [B1, Proposition II.2.11], any weak equivalence $\hat{g}: U \rightarrow V$ induces a bijection $\hat{g}_{*}:\left[I^{n}, U\right]^{u} \rightarrow\left[I^{n}, V\right]^{\hat{g} u}$. Note that given a map of $(n-1)$-th order chain complexes $g: K^{(n-1)} \rightarrow R^{(n-1)}$, an extension of $R^{(n-1)}$ to an $n$-th order resolution $R$ is determined by a choice of $n$-tracks for $R$, and these are precisely elements in sets of the form $\left[I^{n}, V\right]^{\hat{g} u}$ for various mapping spaces $U$ for $K$ and $V$ for $L$. Therefore, $g_{*}$ induces a unique $n$-th order chain complex structure $R$ extending $R^{(n-1)}$.

Similarly for $f: L^{(n-1)} \rightarrow K^{(n-1)}$.

16.19. Lemma. Let $q: \operatorname{nul}_{n} \mathbf{C} \rightarrow \mathrm{Nul}_{n} \mathbf{C}$ be the quotient map of Section 2 and let $q K$ and $q L$ be $n$-th order resolutions of $X$ in $\operatorname{Nul}_{n} \mathbf{C}$. If $q K \sim q L$ are weakly equivalent, then also $K \sim L$ are weakly equivalent over $X$ in $\operatorname{nul}_{n} \mathbf{C}$.

16.20. Lemma. Let $K$ be an $n$-th order resolution of $X$ in $\operatorname{Nul}_{n} \mathbf{C}$ for some $n \geq 2$, and assume that its $(n-1)$-skeleton $K^{(n-1)} \quad\left(\right.$ in $\left.\operatorname{nul}_{n} \mathbf{C}\right)$ is $(N-1)$-(strict, fibrant, exact), for some $N \geq 1$. Then there is a weakly equivalent $n$-th order resolution $L \sim K$ of $X$ which is $N$-(strict, fibrant, exact). 
Proof. Since the $(n-1)$-skeleton of $K$ is strict, the $n$-track $K\left(i, I_{n}\right)$ is given by elements

$$
\alpha_{i} \in \operatorname{Hom}_{\mathbf{A}}\left(\Sigma^{n} K_{i}, K_{i-n-1}\right) \quad i-n-1 \geq-1
$$

for each $2 \leq i \leq N$. We shall show by induction on $0<i \leq N+1$ that $K$ is weakly equivalent to an $n$-th order resolution which is $(i-1)$-(strict, fibrant, exact), so $\alpha_{j}=0$ for $j<i$.

By the obstruction property of $K$ we have $\delta \alpha_{i} \pm \alpha_{i-1} \delta=0$. Then $\delta \alpha_{i}=0$, and the exactness yields $\beta$ with $\alpha_{i}=\beta \delta$. We construct weak equivalences $L \rightarrow R \leftarrow K$ in $\mathrm{Nul}_{n} \mathbf{C}$ which in dimension 0 are given by the commutative diagram

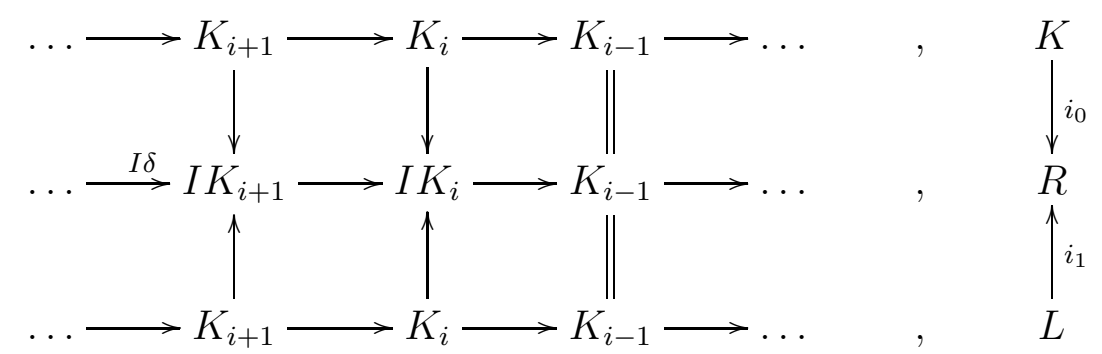

The $(n-2)$-skeleton of $R$ is strict. We define $R\left(i, I_{n-1}\right)$ by $\beta$. Then we can choose $R\left(i, I_{n}\right)$ such that $i_{0}$ is a well-defined map and $L$ is $i$-strict in $\operatorname{Nul}_{n} \mathbf{C}$.

Proof of Theorem 16.7 for $n \geq 2$. By induction on $n$, we assume that the Theorem holds for $n-1$. Let $K$ be a resolution of $X$ in $\mathrm{Nul}_{n} \mathbf{C}$, and let $K^{(n-1)}$ be the $(n-1)$-skeleton of $K$ in $\operatorname{nul}_{n} \mathbf{C}$. For $q K^{(n-1)}$ we get by assumption a weak equivalence $q K^{(n-1)} \sim q L^{(n-1)}$, where $L$ is $N$-strict. Hence by Lemma 16.19 we have $K^{(n-1)} \sim L^{(n-1)}$, and by the Transport Lemma 16.18 we get $K \sim L$ in $\operatorname{Nul}_{n} \mathbf{C}$, where $L^{(n-1)}$ is strict. Now Lemma 16.20 yields $L \sim L^{\prime}$ in $\operatorname{Nul}_{n} \mathbf{C}$, where $L^{\prime}$ is $N$-strict.

Proof of Theorem 15.9. Let $K$ and $L$ be two resolutions of $X$ in $\operatorname{Nul}_{n}$ C. By Theorem 16.7 we have $L \sim L^{\prime}$ and $K \sim K^{\prime}$, where $L^{\prime}$ and $K^{\prime}$ are $N$-(strict, fibrant, exact) for large $N$. This yields a map of spectral sequences $E_{K} \rightarrow E_{L}$ which induces an isomorphism on the $E_{2}$-term. Hence $E_{K} \rightarrow E_{L}$ is also an isomorphism.

Proof of Theorem 15.11. An $N$-strict $N$-fibrant $N$-exact coresolution of $X$ for large $N$, as in Remark [16.8, corresponds to the $X$-coaugmented sequence in [BJ2, §6.7] given by the Adams fiber tower [BJ2, 7.1].

16.21. Remark. Strictification results for $\infty$-homotopy commutative diagrams appear in [BV, Theorem IV.4.37] and [DKS, Theorem 2.4], inter alia. However, these do not yield the precise case needed for Theorem 16.7. The explicit construction given in this context may be of independent interest.

\section{The SECONDARY Ext-GRouP}

We now illustrate the general theory described above in the first interesting case, namely, the secondary Ext-group which is the $E_{3}$-term of the Adams spectral sequence. This is determined by the $d_{2}$-differential, which we now describe. The actual computation of this differential appears in [B2, BJ2, BJ3]. As a test for the theory, 
computer calculations based on those results were carried out, recovering the values of $d_{2}$ in [R, App. 3].

We first specialize Definition 15.8 to the case $m=2$ : let $\mathbf{T}(\leq 1)$ be a $\Sigma$-algebra of left cubical balls and let $K$ be a resolution in $\mathbf{T}(1)$ of $X$, based on $A_{\bullet}$ in $\mathbf{A}$, see (15.3). Then

is given as follows:

$$
d_{2}: \operatorname{Ext}_{\mathbf{A}}^{r}(X, Y) \rightarrow \operatorname{Ext}_{\mathbf{A}}^{r+2}(\Sigma X, Y)
$$

For $\{\beta\} \in \operatorname{Ext}_{\mathbf{A}}^{r}(X, Y)$ with $\beta: A_{r} \rightarrow Y$ in $\mathbf{A}$, we have $\beta \delta_{r+1}=0$, so that there is a 1-track $H$ with $\partial^{1} H=\beta \delta_{r+1}$. On the other hand $K$ yields a 1-track $G$ with $\partial^{1} G=\delta_{r+1} \delta_{r+2}$. Then the obstruction of Definition 15.8 is

$$
\omega=\mathcal{O} \hat{L}\left(r+2, \partial I_{2}\right)=\mathcal{O}\left(H \delta_{r+2}, \beta G\right) \in\left[\Sigma A_{r+2}, Y\right]
$$

and this element represents $d_{2}\{\beta\}=\{\omega\}$.

17.2. Lemma. The differential $d_{2}$ is well defined.

Proof. We first check that $\omega$ is a cocycle. In fact,

$$
\begin{aligned}
\omega\left(\Sigma \delta_{r+3}\right) & =\mathcal{O}\left(H \delta_{r+2}, \beta G\right)\left(\Sigma \delta_{r+3}\right) \\
& =\mathcal{O}\left(H \delta_{r+2} \delta_{r+3}, \beta G \delta_{r+3}\right) \\
& =\mathcal{O}\left(H \delta_{r+2} \delta_{r+3}, \beta \delta_{r+1} G^{\prime}\right) \\
& =0 .
\end{aligned}
$$

Here (1) holds by naturality of $\mathcal{O}$ (see (11.2)). Moreover, $G^{\prime}$ in (2) is the 1-track with $\partial^{1} G^{\prime}=\delta_{r+2} \delta_{r+3}$ given by the resolution $K$, so that $\mathcal{O}\left(G \delta_{r+3}, \delta_{r+1} G^{\prime}\right)=0$. Hence by naturality also $\mathcal{O}\left(\beta G \delta_{r+3}, \beta \delta_{r+1} G^{\prime}\right)=0$, so that (2) holds by the complement rule in Lemma 10.6. Finally (3) holds by the triviality rule.

Next, we show that $\{\omega\}$ does not depend on the choice of $H$. If we choose $H^{\prime}$ instead, there is an $\alpha$ with $H^{\prime}=H+\alpha$, and we get

$$
\omega^{\prime}=\mathcal{O}\left((H+\alpha) \delta_{r+2}, \beta G\right)=\omega \pm \alpha \delta_{r+2}
$$

by the action rule. Hence $\omega-\omega^{\prime}$ is a coboundary, so that $\left\{\omega^{\prime}\right\}=\{\omega\}$.

Finally, we check that $d_{2}\{\beta\}$ is trivial if $\beta$ is a coboundary - that is, $\beta=\beta^{\prime} \delta_{r}$. In fact, we can then choose $H$ to be the 1 -track $\beta^{\prime} G^{\prime \prime}$, where $G^{\prime \prime}$ with $\delta^{1} G^{\prime \prime}=\delta_{r} \delta_{r+1}$ is given by $K$, so that $\mathcal{O}\left(G^{\prime \prime} \delta_{r+2}, \delta_{r} G\right)=0$. Hence also $\mathcal{O}\left(\beta^{\prime} G^{\prime \prime} \delta_{r+2}, \beta^{\prime} \delta_{r} G\right)=0$, so that $\mathcal{O}\left(H \delta_{r+2}, \beta G\right)=0$.

The Lemma is proved in [BJ2] in the context of track categories, above we use only algebras of left 1 -cubical balls. The proof that $d_{2} d_{2}=0$ requires the product rule below.

Next, we prove that the assumption on $L$ in Definition 15.8 is satisfied for $m=2$. This leads to the definition of the differential $d_{3}$.

17.3. Lemma. Suppose that $d_{2}\{\beta\}=0$. Then for $m=2$ there is a chain complex $L$ as in Definition 15.8.

Proof. The assumption $d_{2}\{\beta\}=0$ shows that $\omega=\mathcal{O}\left(H \delta_{r+2}, \beta G\right)=\alpha \delta_{r+2} \quad$ is a coboundary. Hence we get by the action rule $H^{\prime}=H \pm \alpha$, so that $\mathcal{O}\left(H^{\prime} \delta_{r+2}, \beta G\right)=0$. Hence we define the chain complex $L$ by $H^{\prime}$ and by $K$. 
In the context of a $\Sigma$-track algebra $\mathbf{T}(\leq n) \quad(n \geq 1)$, the following result can be proved which is the higher dimensional analogue of Lemma 17.2,

17.4. Proposition. Given L, $\hat{L}$, and

$$
\omega=\mathcal{O} \hat{L}\left(r+m+1, \partial I_{m+1}\right)
$$

as in Definition 15.8, then $\omega$ is a cocycle, that is:

$$
\omega\left(\Sigma^{m} \delta_{r+m+2}\right)=0 .
$$

Moreover, if $\beta$ is a coboundary, then $L$ and $\hat{L}$ can be chosen so that $\omega=0$. Let $L$ be given and let $\hat{L}$ and $\bar{L}$ be two choices as in Definition 15.8. Then $\bar{\omega}:=\mathcal{O} \bar{L}(r+m+$ $\left.1, \partial I_{m+1}\right) \quad$ and $\omega$ differ by a coboundary - that is, $\omega-\bar{\omega}=\alpha\left(\Sigma^{m} \delta_{r+m+1}\right)$.

\section{Appendix: Complete algebras of left Cubical Balls}

Definition 15.8 of the differential $d_{m+1}$ makes sense in any $\sum$-algebra of left cubical balls $\mathbf{T}(\leq n)$, but we need not have $d_{m+1} \circ d_{m+1}=0$. However, this is automatically satisfied in the topological examples of $\$ 7.3$ (and the differential in the Adams spectral sequence is indeed given by Definition 15.8).

In this appendix, which is not needed for the rest of the paper, we describe (without proof) some properties of $\Sigma$-algebras of left cubical balls which are needed to define the higher Ext-groups. For this purpose we introduce the notion of a complete $\Sigma$-algebra of left cubical balls. An example is provided by the $\Sigma$-algebra of left cubical balls $\mathrm{Nul}_{\leq n} \mathbf{C}$, where $\mathbf{C}$ is a complete $\Sigma$-mapping algebra as in Definition 7.2 .

For such a $\mathbf{C}$, the endofunctor $\Sigma: \mathbf{C} \longrightarrow \mathbf{C}$ induces an endofunctor

$$
\Sigma: \mathrm{Nul}_{\leq n} \mathbf{C} \longrightarrow \mathrm{Nul}_{\leq n} \mathbf{C}
$$

of $\Sigma$-algebras of left cubical balls satisfying

$$
\Sigma \mathcal{O}_{B}\left(b_{1}, \ldots, b_{k}\right)=\mathcal{O}_{B}\left(\Sigma b_{1}, \ldots, \Sigma b_{k}\right)
$$

for each left cubical ball of dimension $\leq n$, see 11.1)(3).

18.3. Definition. Let $\mathbf{T}(\leq n)$ be a $\Sigma$-track algebra and let

$$
\Sigma: \mathbf{T}(\leq n) \longrightarrow \mathbf{T}(\leq n)
$$

be an endofunctor of $\mathbf{T}(\leq n)$, as in (18.1), satisfying (18.2), such that $\Sigma$ induces the endofunctor $\Sigma: \mathbf{A} \longrightarrow \mathbf{A}$ of the $\Sigma$-algebra $\mathbf{A}$. Then $\mathbf{T}(\leq n)$ is a complete $\Sigma$-algebra of left cubical balls if the sum rule and the product rule below are satisfied.

18.4. Definition (sum rule). Let $m \leq n$. Assume given a pre-chain complex $L$ in $\mathbf{T}(m)$ based on

$$
Y \stackrel{\alpha}{\longleftarrow} A_{0} \stackrel{\delta_{1}}{\longleftarrow} A_{1} \longleftarrow \ldots \longleftarrow A_{m+1}
$$

and a pre-chain complex $L^{\prime}$ in $\mathbf{T}(m-1)$ based on

$$
Y \stackrel{\beta}{\longleftarrow} \Sigma A_{1} \stackrel{\Sigma \delta_{2}}{\longleftarrow} \Sigma A_{2} \longleftarrow \ldots \longleftarrow \Sigma A_{m+1}
$$


such that $L^{\prime}$ restricted to $\mathbb{Z}(m+1,1)$ coincides with $\Sigma L$. Under these assumptions, the sum rule in dimension $m$ demands that there exist a pre-chain complex $L^{\prime \prime}$ in $\mathbf{T}(m)$ based on (1) such that $L^{\prime \prime}$ restricted to $\mathbb{Z}(m+1,0)$ coincides with $L$ and

$$
\mathcal{O} L^{\prime \prime}\left(m+1, \partial I_{m+1}\right)=\mathcal{O} L\left(m+1, \partial I_{m+1}\right)+\mathcal{O} L^{\prime}\left(m+1, \partial I_{m}\right) .
$$

18.5. Proposition. The sum rule is satisfied in $\mathrm{Nul}_{\leq n} \mathbf{C}$ in (18.1).

Proof. Let $I \approx[0,2]=I \cup I$ be the homeomorphism of intervals carrying $t \in I$ to $2 t$. Then we have:

$$
I^{k+1}=I \times I^{k} \approx(I \cup I) \times I^{k}=I^{k+1} \cup I^{k+1} .
$$

for each $k \geq 0$.

For each $j=1, \ldots, m+1, \quad L^{\prime} \quad$ yields the left $(j-1)$-cube $a_{j}=L^{\prime}\left(j+1, I_{j-1}\right)$ in $\operatorname{Mor}_{\mathbf{C}}\left(\Sigma A_{j+1}, Y\right)$, which by $\tau_{\Sigma}$ in Definition 7.2 yields the $j$-cube $\bar{a}_{j}$ in $\operatorname{Mor}_{\mathbf{C}}\left(A_{j+1}, Y\right)$ adjoint to $\tau_{\Sigma} a_{j}$. Using (1), we define the $j$ cube

$$
L^{\prime \prime}\left(j+1, I_{j}\right)=L\left(j+1, I_{j}\right) \cup \bar{a}_{j} .
$$

This defines $L^{\prime \prime}$ completely, since $L^{\prime \prime}$ restricted to $\mathbb{Z}(m+1,0)$ coincides with $L$. One can now check that the sum formula (18.4) (3) holds.

Let $\mathbf{T}$ be an $(n+k)$-category enriched in left cubical $(n+k)$-sets and let $\mathbf{T}^{n}$ be the $n$-skeleton of $\mathbf{T}$. Then $\mathbf{T}^{n}$ is an $n$-graded category enriched in $n$-cubical sets. We consider a pre-chain complex

$$
R: \mathbb{Z}(\infty, 0)_{\otimes}^{n} \longrightarrow \mathbf{T}^{n}
$$

18.6. Definition. A chain module with operators in $R$ is a functor $L$ which carries a morphism $V: i \longrightarrow-1 \quad(i \geq 0) \quad$ in $\mathbb{Z}(\infty,-1)_{\otimes}^{n}$ to an element

$$
L(V) \in \operatorname{Mor}_{\mathbf{T}}(R, Y)_{\operatorname{dim}(V)+k}
$$

such that the inclusion property

$$
L(V)=\left(d_{V, W} \otimes I^{k}\right)^{*} L(W)
$$

holds if $V$ is in the boundary of $W$ and such that for a composite $V \otimes V^{\prime}$ of morphisms in $\mathbb{Z}(\infty,-1)_{\otimes}^{n}$ the equation

$$
L\left(V \otimes V^{\prime}\right)=L(V) R\left(V^{\prime}\right)
$$

holds, where the right hand side denotes the composite in the $(n+k)$-category $\mathbf{T}$.

18.7. Lemma. A chain module $L$ with operators in $R$ is determined by the elements

$$
L\left(m, I_{m}\right) \in \operatorname{Mor}_{\mathbf{T}}\left(R_{m}, Y\right)_{m+k}
$$

where $I_{0}=\emptyset$ and $m=0, \ldots, n$.

Now let $B=B_{1} \cup \ldots \cup B_{s}$ be a left cubical ball of dimension $k$ with cells $B_{i}$ and gluing maps $d_{e, i}$ as in Definition 9.3. An $s$-tuple $\left(L_{1}, \ldots, L_{s}\right)$ of chain modules $L_{i}$ with operators in $R$ satisfies the gluing condition in $B$ if for $V: m \rightarrow-1$ we have

$$
\left(I^{\operatorname{dim}(V)} \times d_{e, i}\right)^{*} L_{i}(V)=\left(I^{\operatorname{dim}(V)} \times d_{e, j}\right)^{*} L_{j}(V) .
$$

The left cubical ball $C=T_{0}^{n}$ has cells $C_{1}, \ldots, C_{n+1}$. The product $B \times C$ is a left cubical ball with cells $B_{i} \times C_{j}$. Let $\left(c_{1}, \ldots, c_{n+2}\right)=\partial I_{n+1}$, see (4.6). 
18.9. Lemma. Given $\left(L_{1}, \ldots L_{s}\right)$ as in (18.8) we obtain the tuple of $(m+k)$-cubes $(r=n+1) \quad L_{i}\left(r, c_{j}\right) \quad$ satisfying the gluing condition in $B \times C$, so that the obstruction

$$
\mathcal{O}_{B \times C}\left(L_{i}\left(r, c_{j}\right), \quad i=1, \ldots, s \text { and } j=1, \ldots, n+1\right) \text { in } \operatorname{Hom}_{\mathbf{A}}\left(\sum^{n+k} R_{r}, Y\right)
$$

is defined in the $\Sigma$-track algebra $\mathbf{T}=\mathbf{T}(n+k)$. Also the tuple $L_{i}(0, \emptyset)$ satisfies the gluing condition, so that the obstruction

$$
\mathcal{O}_{B}\left(L_{1}(0, \emptyset), \ldots, L_{s}(0, \emptyset)\right) \in \operatorname{Hom}_{\mathbf{A}}\left(\Sigma^{k} R_{0}, Y\right)
$$

is defined in $\mathbf{T}(k)$.

18.10. Definition (product rule). Let $R$ and $L_{1}, \ldots, L_{s}$ be given as above where $R$ is based on

$$
\ldots \longrightarrow R_{n+1} \longrightarrow R_{n} \longrightarrow . . \delta_{1} R_{0} .
$$

Let $\alpha \in \operatorname{Hom}_{\mathbf{A}}\left(\Sigma^{k} R_{0}, Y\right)$ be given by $\mathcal{O}_{B}\left(L_{i}(0, \emptyset)\right)$. Then there exists a pre-chain complex $L^{\prime}$ based on

$$
\Sigma^{k} R_{n+1} \longrightarrow \Sigma^{k} R_{n} \longrightarrow \ldots \stackrel{\Sigma^{k} \delta_{1}}{\longrightarrow} \Sigma^{k} R_{0} \stackrel{\alpha}{\longrightarrow} Y
$$

such that the equation

$$
\mathcal{O}_{C}\left(L^{\prime}\left(n+1, \partial I_{n+1}\right)\right)=\mathcal{O}_{B \times C}\left(L_{i}\left(0, c_{j}\right)\right)
$$

holds in $\operatorname{Hom}_{\mathbf{A}}\left(\sum^{k+n} R_{n+1}, Y\right)$, and $L^{\prime}$ restricted to $\mathbb{Z}(n+1,0)$ coincides with $\Sigma^{k} R$. This is the product rule in $\mathbf{T}(n+k)$.

18.11. Proposition. Let $\mathbf{C}$ be a complete $\Sigma$-mapping algebra. Then $\mathrm{Nul}_{\leq(n+k)}(\mathbf{C})$ satisfies the product rule.

Proof. We have the homeomorphism $S^{k}=B / \partial B$, so we can replace $\Sigma^{k} R_{i}$ by $(B / \partial B) \wedge R_{i}$. Gluing the various $L_{i}$ yields $L^{\prime}$.

18.12. Remark. If $\mathbf{T}(\leq 2 n)$ is a complete $\Sigma$-algebra of left cubical balls (Definition 18.3) the higher Ext-groups $E_{m}^{r, s}$ are well-defined by Definition 15.8 for $m=2, \ldots, n+$ 2. A proof can be given along the lines of the argument given below to show that $d_{2} d_{2}=0$.

Since a complete $\Sigma$-mapping algebra $\mathbf{C}$ yields a complete $\Sigma$-track algebra $\mathrm{Nul}_{\leq 2 n}(\mathbf{C})$, the higher Ext-groups are well defined in this case.

18.13. Example. As an application of the product rule, we prove that $d_{2} d_{2}=0$ : by (15.4) we have a diagram

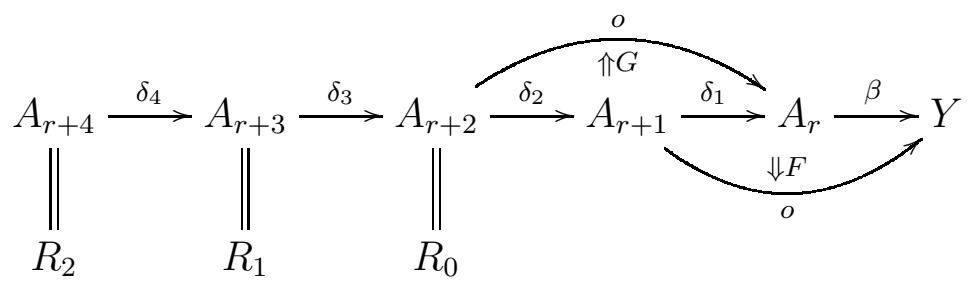

For $B=T_{0}^{1}=B_{1} \cup B_{2}$, we choose $L_{1}(0, \emptyset)=F \delta_{2} \quad$ and $L_{2}(0, \emptyset)=\beta G$, where $F$ and $G$ are 1 -cubes with $G=K\left(r+2, I_{1}\right)$ given by the resolution $K$. Then

$$
\alpha=d_{2} \beta=\mathcal{O}_{B}\left(L_{1}(0, \emptyset), L_{2}(0, \emptyset)\right) \text {. }
$$


Now let $C=T_{0}^{1}=C_{1} \cup C_{2}$ and $\left(c_{1}, c_{2}\right)=\left(I_{1} \otimes \emptyset, \emptyset \otimes I_{1}\right)$. Then $L_{i}\left(2, c_{j}\right)$ is defined as follows:

$$
\begin{array}{llll}
L_{1}\left(2, c_{1}\right)=L_{1}\left(2, I_{1} \otimes \emptyset\right) & = & F K\left(r+3, I_{1}\right) \delta_{4} \\
L_{1}\left(2, c_{2}\right)=L_{1}\left(2, \emptyset \otimes I_{1}\right)= & F \delta_{2} K\left(r+4, I_{1}\right) \\
L_{2}\left(2, c_{1}\right)=L_{2}\left(2, I_{1} \otimes \emptyset\right)= & \beta K\left(r+3, I_{2}\right) \delta_{4} \\
L_{2}\left(2, c_{2}\right)=L_{2}\left(2, \emptyset \otimes I_{1}\right)= & \beta K\left(r+2, I_{1}\right) K\left(r+4, I_{1}\right)
\end{array}
$$

Now the product rule shows that

$$
d_{2} d_{2} \beta=d_{2} \alpha=\mathcal{O}_{B \times C}\left(L_{i}\left(0, c_{j}\right)\right)=0
$$

and the rules in an algebra of left 2-cubical balls show that this obstruction is trivial. In fact, we have

$$
\begin{aligned}
& \mathcal{O}\left(I_{1} I_{1} \emptyset, \emptyset I_{2} \emptyset, I_{1} \emptyset I_{1}, \emptyset I_{1} I_{1}\right) \\
= & \mathcal{O}\left(I_{1} I_{1} \emptyset, \emptyset \emptyset I_{2}, I_{1} \emptyset I_{1}\right) \\
= & 0
\end{aligned}
$$

Here (3) is the obstruction (2), (4) is a consequence of the complement rule, and

$$
\mathcal{O}\left(\emptyset I_{2}, I_{1} I_{1}, I_{2} \emptyset\right)=0
$$

which follows from the fact that $K$ is a resolution. Naturality yields

$$
\mathcal{O}\left(\emptyset \emptyset I_{2}, \emptyset I_{1} I_{1}, \emptyset I_{2} \emptyset\right)=0
$$

Finally, (5) follows from the triviality rule.

\section{REFERENCES}

[A] J.F. Adams, "On the structure and applications of the Steenrod algebra", Comm. Math. Helv. 32 (1958), 180-214.

[B1] H.-J. Baues, Algebraic homotopy, Cambridge studies in Advanced Math. 15, Cambridge 1989.

[B2] H.-J. Baues, The Algebra of secondary cohomology operations, Progress in Math. 247, Birkhäuser, 2006

[B3] H.-J. Baues, "Higher order track categories and the algebra of higher order cohomology operations", Georgian Math. J. 17 (2010), 25-55.

[BB1] H.-J. Baues \& D. Blanc "Comparing cohomology obstructions", J. Pure \& Appl. Alg. 215 (2011), 1420-1439.

[BB2] H.-J. Baues \& D. Blanc "Stems and Spectral Sequences", Alg. \& Geom. Top., 10 (2010), pp. 2061-2078.

[BD] H.-J. Baues \& W. Dreckmann, "The cohomology of homotopy categories and the general linear group", K-Theory 3 (1989), 307-338.

$[\mathrm{BaF}] \quad$ H.-J. Baues \& M. Frankland, in preparation.

[BJ1] H.-J. Baues \& M. Jibladze, "Classification of abelian track categories", K-Theory 25 (2002), 299-311

[BJ2] H.-J. Baues \& M. Jibladze, "Secondary derived functors and the Adams spectral sequence", Topology 45 (2006), 295-324.

[BJ3] H.-J. Baues \& M.A. Jibladze, "Dualization of the Hopf algebra of secondary cohomology operations and the Adams spectral sequence", J. K-Theory 7 (2011), pp. 203-347.

[BW] H.-J. Baues \& G. Wirsching, "Cohomology of small categories", J. Pure Appl. Alg. 38 (1985), 187-211

[BJT] D. Blanc, M.W. Johnson, \& J.M. Turner, "Higher homotopy operations and cohomology", J. K-Theory 5 (2010), 167-200. 
[Bo] J.M. Boardman, "Homotopy Structures and the Language of Trees",", Algebraic Topology (Univ. Wisconsin, Madison, WI, 1970), Proc. Symp. Pure Math. 22, AMS, Providence, RI, 1971, 37-58.

[BV] J.M. Boardman \& R.M. Vogt, Homotopy Invariant Algebraic Structures on Topological Spaces, Springer-Verlag Lec. Notes Math. 347, Berlin-New York, 1973.

$[\mathrm{BoF}] \quad$ A.K. Bousfield \& E.M. Friedlander, "Homotopy theory of $\Gamma$-spaces, spectra, and bisimplicial sets", in M.G. Barratt \& M.E. Mahowald, eds., Geometric Applications of Homotopy Theory, II Springer-Verlag Lec. Notes Math. 658, Berlin-New York, 1978, 80-130

[C] D.-C. Cisinski, "Les préfaisceaux comme modèles des types d'homotopie", Astérisque 308, Soc. Math. France, Paris, 2006.

[DKS] W.G. Dwyer, D.M. Kan, \& J.H. Smith, "Homotopy commutative diagrams and their realizations", J. Pure 83 Appl. Alg., 57 (1989), 5-24.

[EKMM] A.D. Elmendorf, I. Křiž, M.A. Mandell, \& J.P. May, Rings, modules, and algebras in stable homotopy theory, AMS, Providence, RI, 1997.

[HSS] M.A. Hovey, B.E. Shipley, J.H. Smith, "Symmetric spectra", J. AMS 13 (2000), 149-208

[I] S.B. Isaacson, "Symmetric Cubical Sets", J. Pure Appl. Alg. 215 (2011), 1146-1173.

$[\mathrm{J}] \quad$ J.F. Jardine, "Categorical homotopy theory", Homology, Homotopy \& Applic. 8 (2006), 71-144.

[K1] L. Kristensen, "On secondary cohomology operations", Math. Scand. 12 (1963), 57-82.

[K2] L. Kristensen, "On a Cartan formula for secondary cohomology operations", Math. Scand. 16 (1965), 97-115.

[MMSS] M.A. Mandell, J.P. May, S. Schwede, \& B. Shipley, "Model categories of diagram spectra", Proc. London Math. Soc. (3) 82 (2001), 441-512.

[M] J.R. Munkres, "The special homotopy addition theorem", Mich. Math. J, 2 (1953/54), 127-134.

[P] T. Pirashvili, " $H^{3}$ and models for the homotopy theory", Trudy Tbilissi 94 (1992), 81-98.

[R] D.C. Ravenel, Complex Cobordism and Stable Homotopy Groups of Spheres, Academic Press, Orlando, 1986.

[S] N.E. Steenrod, "A convenient category of topological spaces", Michigan Math. J, 14 (1967), pp. 133-152.

[V] R.M. Vogt, "Convenient categories of topological spaces for homotopy theory", Arch. Math. (Basel) 22 (1971), pp. 545-555.

[Wa] G. Walker, "Long Toda brackets", in Proceedings of the Advanced Study Institute on Algebraic Topology, Vol. III (Aarhus, 1970), Aarhus, 1970, 612-631.

[Wh] G.W. Whitehead, Homotopy Theory, MIT Press, Cambridge, MA, 1966.

Max-Planck-Institut für Mathematik, Vivatsgasse 7, 53111 Bonn, Germany

E-mail address: baues@mpim-bonn.mpg.de

Department of Mathematics, University of Haifa, 3498838 Haifa, Israel

E-mail address: blanc@math.haifa.ac.il 